\title{
A new method for extending solutions to the self-similar relativistic magnetohydrodynamics equations for black hole outflows
}

\author{
Ceccobello ${ }^{1 \star}$ C., Cavecchi ${ }^{2,3}$ Y., Heemskerk ${ }^{1}$ M.H.M., Markoff ${ }^{1}$ S., Polko ${ }^{4}$ P., Meier ${ }^{5}$ D. \\ 1 "Anton Pannekoek" Instituut voor Sterrekunde, Universiteit van Amsterdam, Science Park 904, 1098 XH Amsterdam, The Netherlands \\ 2 Department of Astrophysical Sciences, Princeton University, Peyton Hall, Princeton, NJ 08544, USA \\ 3 Mathematical Sciences and STAG Research Centre, University of Southampton, SO17 1BJ, UK \\ ${ }^{4}$ University of Maryland at College Park, Dept. of Physics, Joint Space-Science Institute, \\ 3114 Physical Sciences Complex, College Park, MD 20742, USA \\ 5 Jet Propulsion Laboratory, California Institute of Technology, Pasadena, CA 91109, USA
}

3 July 2018

\begin{abstract}
The paradigm in which magnetic fields play a crucial role in launching/collimating outflows in many astrophysical objects continues to gain support. However, semianalytical models including the effect of magnetic fields on the dynamics and morphology of jets are still missing due to the intrinsic difficulties in integrating the equations describing a collimated, relativistic flow in the presence of gravity. Only few solutions have been found so far, due to the highly nonlinear character of the equations together with the need to blindly search for singularities. These numerical problems prevented a full exploration of the parameter space. We present a new integration scheme to solve $r$-self-similar, stationary, axisymmetric magnetohydrodynamics equations describing collimated, relativistic outflows crossing smoothly all the singular points (Alfvén point and modified slow/fast points). For the first time, we are able to integrate from the disk mid-plane to downstream of the modified fast point. We discuss an ensemble of jet solutions, emphasising trends and features that can be compared to observables. We present, for the first time with a semi-analytical MHD model, solutions showing counter-rotation of the jet for a substantial fraction of its extent. We find diverse jet configurations with bulk Lorentz factors up to 10 and potential sites for recollimation between $10^{3}-10^{7}$ gravitational radii. Such extended coverage of the intervals of quantities, such as magnetic-to-thermal energy ratios at the base or the heights/widths of the recollimation region, makes our solutions suitable for application to many different systems where jets are launched.
\end{abstract}

Key words: black hole physics - magnetohydrodynamics (MHD) - galaxies: jets stars: jets - methods: numerical

\section{INTRODUCTION}

Since their discovery, relativistic collimated outflows of matter have been observed in many astrophysical objects and they are known to be associated with accretion flows. Jets reveal themselves at different scales and redshifts, showing an extreme diversity in energetics, shapes and emission. Jets are found to be characteristic features of black hole systems, such as X-ray binaries (XRBs) and active galactic nuclei (AGN), as well as of young stellar objects (YSOs), explosive transients such as tidal disruption events (TDEs) and

* Contact e-mail: c.ceccobello@uva.nl gamma-ray bursts (GRBs). Observations suggest that jets are an energetically important component of the system that hosts them, because the jet power appears to be comparable to the accretion power (see e.g. Rawlings \& Saunders 1991; Nemmen \& Tchekhovskoy 2015, for a more recent discussion). Significant evidence has been found of the effect of jets not only on the immediate proximity of the central object, but also on their surrounding environment, where they deposit the energy extracted from the accretion flow (e.g. Gallo et al. 2005; Fabian 2012). To launch, accelerate and collimate a relativistic outflow over such large distances, magnetic fields need to be invoked. Observational evidence, such as polarization measurements both in the radio (Martí- 
Vidal et al. 2015) and in the hard X-rays (Laurent et al. 2011), support the idea that ordered magnetic fields are a key ingredient in the jet phenomena, and have a significant effect on their emission as well. Understanding what causes the jet to be launched from an accretion disk, which mechanisms determine its shape and extension and where the radiation is produced in these systems is one of the most fundamental questions in astrophysics and needs to be addressed promptly.

Multiwavelength continuum emission from jets and disks is observed for all accreting, jet-launching sources, XRBs in particular can provide essential pieces of information because extensive monitoring of these sources shows that they go through a duty cycle multiple times during their lives (Fender et al. 2004). During an outburst, they spend most of their time in the low-luminosity hard state where they exhibit a mildly relativistic steady-state jet launched from a likely recessed disk. In this state, the jet dominates the total power and shows a characteristic power-law spectrum that may extend to high energies. Many outbursts show a rapid increase in luminosity, bringing the system close to its Eddington limit. The standard paradigm has the disk inner radius moving closer to the black hole while the jet becomes ballistic, emitting superluminal knots. Eventually the jet switches off and the emission is dominated by the disk. The spectrum becomes softer and loses its nonthermal high-energy component (high-luminosity soft state). Finally, the system slowly decays into a hard state and the cycle restarts (see, e.g. Belloni \& Motta 2016, for a recent review). When the emission is dominated by the compact steady-state jet, its characteristic synchrotron emission can span several orders of magnitude in frequency. At the wavelength range where the synchrotron transitions from the optically thin $(\tau<1)$ to the optically thick regime $(\tau>1)$, the spectrum shows a break and it becomes a flat/inverted power-law, characteristic of the self-absorbed synchrotron (see Romero et al. 2017, for a recent review). The region in the jet corresponding to the break frequency is believed to be the site where particles are first accelerated (Markoff 2010), potentially by internal shocks (Malzac 2014). The jet break can be inferred from observations to occur over a fairly large range of distances from the black holes $\sim 10-10^{4}$ gravitational radii (hereafter $r_{\mathrm{g}}$ ), and it has been seen to span 4 orders of magnitude in frequency during a state transition in a single object (Russell et al. 2014). Similar spectral features and a duty cycle $10^{7}-10^{8}$ times longer are seen in AGN as well, and a power-unification scenario has been proposed independently by Merloni et al. (2003) and Falcke et al. (2004). They showed that AGN and XRBs of the same relative luminosity, rescaled by the mass of the black hole, can be explained with the same physics framework. While XRBs provide unique constraints on the emission of jets and accretion disks thanks to the multiwavelength monitoring of the activity of the source during state-transition episodes, AGN are ideal for studying the structure of jets and the dynamical processes that shape them.

Indeed, in the case of nearby AGN, high spatial resolution observations are now possible with very long baseline interferometry (VLBI) in both $\mathrm{cm}$ and now mm bands. These data provide unprecedented constraints on the geometry and the dynamics of jets, such as the jet opening angle, the height and the width of knots associated with standing shock fea- tures, such as HST-1 in M87's jet. Recently, high-resolution VLBI observations of M87 by Hada et al. (2016) resolved and imaged the inner core of the galaxy down to $\sim 10 r_{\mathrm{g}}$, revealing the innermost structure of the jet. VLBI/VLBA observations (Asada et al. 2014; Mertens et al. 2016) constrained the bulk Lorentz factor of the jet of M87 to be mildly relativistic, i.e. $\gamma_{\mathrm{j}} \sim 1-3$. In the near future, with the beginning of the Event Horizon Telescope (EHT) era, observations will resolve the nearest black holes (Sagittarius $A *$ and M87) down to the event horizon scale (see e.g. Doeleman et al. 2008). This unprecedented resolution will shed new light on the immediate proximity of black holes, possibly unveiling the jet/disk connection and the mechanisms responsible for the acceleration and collimation in the first stages of jet formation. Finally, Meyer et al. (2013) measured the proper motions of the knots downstream of HST-1 with the Hubble Space Telescope, finding significant evidence of transverse and parallel motion with respect to the jet axis. This is evidence of a helical magnetic field beyond HST-1 and it brings important constraints on the modelling of jets at larger distances from the $\mathrm{BH}$.

Using both XRBs and AGN to obtain insight on the apparently similar jet phenomena is extremely important as demonstrated by the activity in this field of research. However, an adequate modelling of jets, including a detailed treatment of both the radiative processes and the magnetohydrodynamics (MHD), is still far from being achieved.

Thanks to the dramatic improvement of computational power, accretion disks and jets can be modelled with general relativistic magneto-hydrodynamic (GRMHD) simulations (e.g. Koide et al. 2002; McKinney 2006; Hawley \& Krolik 2006; Tchekhovskoy et al. 2011; Tchekhovskoy \& Bromberg 2016). Full 3D simulations allow detailed study of the stability of jets under different sets of initial conditions, and they provide a unique overview of how jets are launched, how the disk and jet interact during this phase and how they approach a stable configuration. However, the lack of crucial ingredients such as non-ideal processes or self-consistent radiative processes makes a direct test against observational data still a challenge. It is, however, worth noting that efforts are currently made to incorporate simplified treatments of electron microphysics in GRMHD simulations (e.g. Ressler et al. 2015; Mościbrodzka et al. 2016).

A complementary method to simulations is given by semi-analytical models for multiwavelength emission from disk/jet systems where the geometry and the dynamics are generally fixed and simplified. Many of such models have been proposed, for instance by Romero et al. (2003); Markoff et al. (2005); Yuan et al. (2005); Potter \& Cotter (2012); Pepe et al. (2015); Zdziarski et al. (2012, 2014) and they have been successful in reproducing the spectral energy distribution of accretion disks and jets. However, the treatment of magnetic fields is also greatly simplified and its orientation is usually not considered. Finally, they all present a certain degree of degeneracy between combinations of input parameters that give statistically equivalent fits to the same data set. Introducing a self-consistent treatment of MHD via semi-analytical models can help reduce the freedom in the parameter space, allowing for better constraining the fits to the observations.

A number of such models have been developed since the 80 s90s, with pioneering works by Blandford \& Payne (1982); 
Lovelace \& Contopoulos (1990); Li et al. (1992); Contopoulos (1994); Sauty \& Tsinganos (1994); Bogovalov \& Tsinganos (1999). The MHD system of equations describing an accelerating flow is a highly nonlinear system which changes nature from elliptical to hyperbolic several times across the interval of integration. Independently of the geometry of the system, it has been shown that three critical surfaces exist in correspondence to such transitions and they determine as well the onset of magnetosonic waves of different type. These are called fast, slow magnetosonic and Alfvén waves. The slow magnetosonic singular surface (SMSS) appears close to the central object and upstream of the Alfvén surface. The fast magnetosonic singular surface (FMSS) is located downstream of the Alfvén point and it is suspected to be linked with a recollimation of the streamlines describing the flow (see e.g. Meier 2012, for a complete discussion and derivation). The FMSS, therefore, could tentatively be identified with the jet break seen in observations of AGN and XRBs, while the properties of the flow at SMSS could instead provide important constraints on the input parameters of radiative transfer models, such as e.g. the magnetic-to-thermal energy ratio at the base and the initial bulk velocity. However, the MHD equations that exhibit all the three singular surfaces must include gravity and the effect of thermal pressure in the total internal energy of the flow to properly describe the region close to the black hole. Moreover, to describe typical astrophysical jets, the equations need to allow the flow to become relativistic. Vlahakis et al. (2000, herefter VTST00) and Vlahakis \& Königl (2003, hereafter VK03) derived the MHD system of equations under the assumption of radial self-similarity, first including a simplified gravity term (kinetic term) and, later removing it, to include relativistic effects. Only recently, Polko et al. (2010, 2013, 2014, hereafter, respectively PMM10, PMM13 and PMM14) derived the equations for a self-similar relativistic MHD flow including enthalpy and gravity, and found solutions crossing smoothly all three singular surfaces. Under the assumption of self-similarity, the singular surfaces are cones and intersect a streamline only in one point. They are usually referred to as "modified" slow and fast points (MSP, MFP) and Alfvén point (AP). We will adopt this terminology from here on. For a more detailed history of the derivation of the model, we address the interested reader to the papers cited above and references therein.

Since only at the AP analytical formulae can be written explicitly, locating the MFP and MSP and performing the integration of the equations through them is not an easy numerical problem to solve. These unknowns add a large degree of complexity to the problem and common integration techniques fail to retrieve solutions in most cases. Although successful in solving such highly nonlinear set of MHD equations, PMM14 were limited in the range of jet solutions that they could retrieve due to their numerical approach. Following VK03, PMM14 developed an algorithm where the integration starts from the Alfvén point and "shoots" towards the other two singular points (MFP and MSP). The Alfvén point can be regularised analytically by using the De L'Hôpital rule on the terms that are in indefinite form $0 / 0$. The other two singular points are later found by extrapolation (for more details about the shooting method see e.g. Press et al. 1993 and Stoer \& Bulirsch 2013). When the equations are integrated towards a singularity, however, the error with respect to the exact solution can be large. As a consequence of the limitations intrinsic to the adopted numerical scheme, PMM14 were limited to a small portion of the parameter space.

In this paper, we present a new method for exploring the parameter space and finding solutions to the MHD equations building on the work of the PMM papers. Using this method, we are able to explore more efficiently a much larger fraction of the parameter space. This will allow the model to be applicable to many other difficult flow solution problems in astrophysics, as well as potentially other fields. Moreover, this class of models can be coupled with fairly accurate radiative transfer models (see Markoff et al. 2005; Maitra et al. 2009; Connors et al. 2016; Crumley et al. 2017), which can handle spectral fitting in a reasonable computational time. This paper follows the following structure: in Section 2 we describe the system of equations that we solve and discuss modifications in the equations compared to PMM14 with respect to their dependence on the gravitational potential. In Section 3 we present our numerical scheme and in Section 4 we compare our results with PMM14. We show that the solutions are extremely sensitive to the gravity terms and when the corrected functions of the gravitational potential are used, the self-similarity assumption is more easily broken. In Section 5 we present a partial study of the parameter space, unaccessible to previous studies. We included the details of our method in a series of Appendixes: in Appendix A we define the equations that we solve in explicit form with the corrected gravity terms and the new Alfvén regularity condition. In Appendix B we describe the derivation of the functions of the pseudo-potential in the gravity terms. In Appendix $\mathrm{C}$ we discuss in detail our approach in finding the locations of the unknown singular points, MFP and MSP, and how we perform the integration. Finally, in Appendix D we give the conversion of the most relevant quantities into physical units.

\section{SET UP OF THE EQUATIONS}

Our goal is to describe an outflow launched from an accretion disk in the presence of a magnetic field by the Blandford-Payne mechanism (Blandford \& Payne 1982). We use cylindrical coordinates $(\varpi, \phi, z)$ and spherical coordinates $(r, \phi, \theta)$ and impose axial symmetry, i.e. $\partial / \partial \phi=0$ and assume the flow to be stationary, $\partial / \partial t=0$. For the electric field $E$, we impose the freeze-in condition, $E=-\mathbf{v} \times \mathbf{B}$, for the Ohm's law in ideal MHD and due to the axial symmetry $E_{\phi}=0$. In what follows we adopt the notation of VK03 and PMM14.

As in VK03, we assume that the dependence of each variable on $r$ and $\theta$ is separable, and that the radial dependence can be expressed as a power law of $r$ (see VK03). This assumption leads to self-similar solutions that obey a system of ordinary differential equations plus algebraic constraints. The four unknowns are the specific relativistic enthalpy $\xi c^{2}$, the poloidal Alfvén Mach number, $M=$ $\left(\gamma V_{\mathrm{p}} / B_{\mathrm{p}}\right)\left(4 \pi \rho_{0} \xi\right)^{1 / 2}\left(V_{\mathrm{p}}\right.$ and $B_{\mathrm{p}}$ are the poloidal components of the velocity and the magnetic field, $\rho_{0}$ is the baryon rest mass density, $\gamma$ is the bulk Lorentz factor), the cylindrical radius $x$ in units of the light cylinder, $x=\varpi \Omega / c(c$ is the speed of light, $\Omega$ is the angular velocity of the flow), 

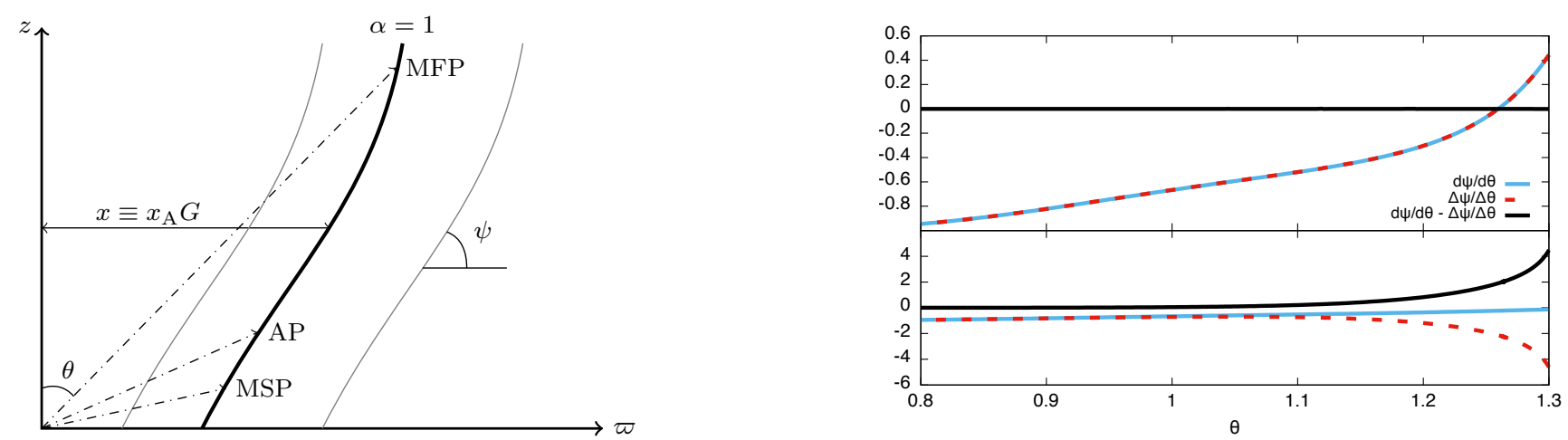

Figure 1. Left panel: System of coordinates we adopt to describe a solution of eq. (1)-(4), which is typically the "reference" streamline identified with the label $\alpha \equiv \varpi_{A}^{2} / \varpi^{2}=1$. The four unknowns in the system (1)-(4), together with all the other quantities, are functions of $\theta$, which is the angle between a point in the streamline and the $z$-axis. The angle that the tangent to the streamline makes with the horizontal axis is $\psi$, while the distance from a point of the streamline to the $z$-axis is defined by its cylindrical radius in units of the light cylinder $x$. Right panel: Discrepancy between the derivative of $\psi$, calculated directly from eq. $5, d \psi / d \theta$, and inferred numerically at each step from eq. $3, \Delta \psi / \Delta \theta$ with the corrected functions of the gravitational potential (top panel) and with the one used by PMM14 (bottom panel). The MSP and the black hole are on the right.

and the angle $\psi$ between the streamline and the horizontal axis. These are functions of $\theta$ only. We further substitute $x=x_{\mathrm{A}} G$, such that for each streamline, $G$ is the cylindrical radius scaled by its value at the Alfvén point.

The system of equations that we solve can be written in the form:

$$
\begin{aligned}
\frac{d M^{2}}{d \theta} & =\frac{B_{2} C_{1}-B_{1} C_{2}}{A_{1} B_{2}-A_{2} B_{1}} \equiv \frac{\mathcal{N}_{1}}{\mathcal{D}}, \\
\frac{d G^{2}}{d \theta} & =\frac{2 G^{2} \cos (\psi)}{\sin (\theta) \cos (\psi+\theta)}, \\
\frac{\mu^{\prime 2}}{\xi^{2}} & \left\{\frac{G^{4}\left(1-M^{2}-x_{\mathrm{A}}^{2}\right)^{2}-x^{2}\left(G^{2}-M^{2}-x^{2}\right)^{2}}{G^{4}\left(1-M^{2}-x^{2}\right)^{2}}\right\} \\
& =1+\frac{F^{2} \sigma_{\mathrm{M}}^{2} M^{4} \sin ^{2} \theta}{\xi^{2} x^{4} \cos ^{2}(\theta+\psi)}, \\
M^{2} & =q \frac{\xi}{(\xi-1)^{1 /(\Gamma-1)}},
\end{aligned}
$$

where the functions $A_{i}, B_{i}, C_{i}$ with $i=1,2$ are defined in Appendix A, while $F$ is a parameter describing the scaling of the magnetic field with respect to the radius as $B \propto r^{F-2}$. $\sigma_{\mathrm{M}} \equiv B_{\mathrm{p}} \Omega^{2} /\left(4 \pi \rho_{0} V_{\mathrm{p}} c^{3}\right)$ is the magnetization parameter introduced by Michel (1969). For more details, see Appendix A, while for a complete derivation of the original equations we refer the reader to VK03 and PMM14. Here we will provide a short description of their meaning and use.

Eq. (1) is the so-called wind equation for the poloidal Alfvén Mach number, $M$, and can be derived from the Euler equation. Eq. (2) describes the evolution of the dimensionless cylindrical radius and can be derived from the Euler equation. Eq. (3) is the Bernoulli equation describing the energy conservation along the poloidal component of the magnetic field line. From this equation we derive $\psi$, once $M^{2}$ and $G^{2}$ are know at each integration step. Finally, Eq. (4) is the relation between the poloidal $M$, the enthalpy $\xi$ and the dimensionless adiabatic parameter $q$ (Tab. 1), which we use to derive $\xi$ at each step.

Our approach differs from that of PMM14 in how the gravity term is treated. Gravity enters through the pseudopotential $P_{\mathrm{g}}$ (see Appendix A). PMM14, following Meier
(2012), considered $P_{\mathrm{g}}$ to be small and therefore approximated terms like $1 /\left(1-P_{\mathrm{g}}\right)$ as $\left(1+P_{\mathrm{g}}\right)$ or used other similar approximations. $P_{\mathrm{g}}$ enters the $C_{i}$ terms in Eq. (1) and the analogous equation for $\psi$

$$
\frac{d \psi}{d \theta}=\frac{A_{1} C_{2}-A_{2} C_{1}}{A_{1} B_{2}-A_{2} B_{1}} \equiv \frac{\mathcal{N}_{2}}{\mathcal{D}},
$$

which in principle we do not use, since we can exploit the much more tractable and accurate Bernoulli equation (Eq. $3)$.

However, we noticed that the rate of change of $\psi$ with respect to $\theta$ as derived from the system of equations (1) - (4) was not consistent with the prediction of Eq. (5), with a substantial discrepancy upstream of the Alfvén point (see right panel of Fig. 1).

The reason can be understood as follows: Meier (2012) obtained the Bernoulli and the transfield equations neglecting terms $\propto P_{\mathrm{g}}^{2}$ (see equations F.16 and F.18 in Meier 2012). Our Eq. (3) corresponds to Meier's equation F.16. In order to obtain Eq. (1), we take derivatives of the Bernoulli equation Eq. (3) and therefore of $P_{\mathrm{g}}$ (see Appendix B). However, we also keep the Bernoulli equation in its original form. If we were to approximate the term $1 /\left(1-P_{\mathrm{g}}\right)$ in the derivatives, the resulting equation would not be consistent with Eq. (3) anymore, because we would obtain a different version of the Bernoulli equation when integrating them back. Of course, this difference would be more pronounced upstream of the Alfvén point where $P_{\mathrm{g}}$ is greater, explaining the discrepancy we measured.

We use Eq. (3) in order to evaluate quickly $\psi$ and therefore we need to retain in $C_{1}$ the full term $1 /\left(1-P_{\mathrm{g}}\right)$ and calculate full derivatives of $P_{\mathrm{g}}$ with respect to $\theta$ when needed ${ }^{1}$. This approach brings back self-consistency between Eqs. (1)(4) and Eq. (5).

Analogously, the gravity correction term to $C_{2}$ comes from derivatives of $P_{\mathrm{g}}$ in the transfield equation written to the same order of approximation as the Bernoulli equation

${ }^{1}$ We use $d P_{\mathrm{g}} / d \theta=\partial P_{\mathrm{g}} / \partial \theta+\partial P_{\mathrm{g}} / \partial G \cdot d G / d \theta$. 
Table 1. Model parameters.

\begin{tabular}{|c|c|c|}
\hline $\begin{array}{l}\text { Input } \\
\text { paran }\end{array}$ & & \\
\hline$F$ & $F \equiv d \log I / d \log \varpi+1$ with $B \propto r^{F-2}$ & determines the shape of the magnetic field at the base \\
\hline$\Gamma$ & $P=Q \rho_{0}^{\Gamma}$ & polytropic index of the gas \\
\hline$\sigma_{\mathrm{M}}$ & $\sigma_{\mathrm{M}} \equiv B_{\mathrm{p}} \Omega^{2} /\left(4 \pi \rho_{0} V_{\mathrm{p}} c^{3}\right)$ & magnetization parameter \\
\hline$\varpi_{\mathrm{A}}$ & $1 / r=\sin \theta /\left(\varpi_{\mathrm{A}} G\right)$ & $\begin{array}{l}\text { Alfvén cylindrical radius, } \\
\text { used to define the gravitational potential }\end{array}$ \\
\hline$\theta_{\mathrm{A}}$ & (see Fig. 1) & angular distance of the Alfvén point from the jet axis \\
\hline$\psi_{\mathrm{A}}$ & (see Fig. 1) & $\begin{array}{l}\text { inclination of the stream line } \\
\text { with respect to the horizontal axis at the AP }\end{array}$ \\
\hline \multicolumn{3}{|c|}{$\begin{array}{l}\text { Fitted } \\
\text { parameters }\end{array}$} \\
\hline$x_{\mathrm{A}}^{2}$ & (see Fig. 1) & square of the Alfvén cylindrical radius in units of light cylinder \\
\hline$q$ & $q=B_{0}^{2} \alpha^{F-2} x_{\mathrm{A}}^{4} /\left(4 \pi c^{2} F^{2} \sigma_{\mathrm{M}}^{2}\right)\left(\Gamma Q /\left(c^{2}(\Gamma-1)\right)\right)^{1 /(\Gamma-1)}$ & dimensionless adiabatic coefficient \\
\hline$\theta_{\mathrm{MFP}}$ & (see Fig. 1) & angular distance of MFP from the jet axis \\
\hline$\theta_{\mathrm{MSP}}$ & (see Fig. 1) & angular distance of MSP from the jet axis \\
\hline
\end{tabular}

(equation F.18 of Meier 2012). For keeping the same consistency as mentioned before, we keep the full terms of the derivatives of $P_{\mathrm{g}}$ in $C_{2}$ as well (Appendix B).

It is worth noting that some of the constants of motions along the streamlines are affected by the inclusion of gravity within a general relativistic formalism as done by PMM14 following Meier (2012). The specific energy $\mu$, defined in VK03 (eq. 13d), is not a constant of motion anymore, while the following one is:

$$
\left(1-P_{\mathrm{g}}\right)[\gamma+\gamma(\xi-1)+S]=\left(1-P_{\mathrm{g}}\right) \mu \equiv \mu^{\prime} .
$$

The first term on the left-hand side is the kinetic energy, the second is the internal energy, the third is the Poynting energy $\left(S=-\varpi \Omega B_{\phi} / \Psi c^{2}\right.$, with $\Psi=4 \pi \rho_{0} \gamma V_{\mathrm{p}} / B_{\mathrm{p}}$ is the mass-to-magnetic flux ratio ${ }^{2}$ ), the multiplicative factor is the contribution of gravity with $P_{\mathrm{g}}$ defined as in eq. (B1). Similarly, the constant specific angular momentum (see VK03, Eq. 13c), becomes now

$$
\left(1-P_{\mathrm{g}}\right)\left[\xi \gamma \varpi V_{\phi}-\frac{\varpi B_{\phi}}{\Psi}\right]=\left(1-P_{\mathrm{g}}\right) L \equiv L^{\prime} .
$$

All the other constants of motion remain unchanged. We can recast Eq. (6) and (7) in the following compact notation:

$$
\begin{aligned}
& \mu^{\prime}=\mu_{\mathrm{HD}}^{\prime}+\mu_{\mathrm{M}}^{\prime}, \\
& L^{\prime}=L_{\mathrm{HD}}^{\prime}+L_{\mathrm{M}}^{\prime},
\end{aligned}
$$

where

$$
\begin{aligned}
\mu_{\mathrm{HD}}^{\prime} & =\left(1-P_{\mathrm{g}}\right) \xi \gamma, & L_{\mathrm{HD}}^{\prime} & =\left(1-P_{\mathrm{g}}\right) \xi \gamma \varpi V_{\phi}, \\
\mu_{\mathrm{M}}^{\prime} & =\left(1-P_{\mathrm{g}}\right) S, & L_{\mathrm{M}}^{\prime} & =\left(1-P_{\mathrm{g}}\right) \varpi B_{\phi} / \Psi,
\end{aligned}
$$

are the hydrodynamical and magnetic components of the total energy and the angular momentum which will be used in Sec. 5.

2 Here we do not use the italic subscript "A" for $\Psi$ to avoid confusion with the roman subscript meaning that a quantity has been calculated at Alfvèn, but it is the equivalent to Eq. 13b in VK03.

\section{NUMERICAL METHOD}

The system of equations (1)-(4) allows for different families of solutions which may or may not cross the singular point(s), similar to the case for hydrodynamic (Parker 1958) or magnetohydrodynamic (Weber \& Davis 1967) solar winds. In the case of the system of equations (1)-(4) and under the assumption of self-similarity, there can be up to three singular points: the Alfvén point AP, the modified fast magnetosonic point MFP and the modified slow magnetosonic point MSP (see Fig. 2). There is only one family of solutions that crosses all the points while it accelerates away from the black hole (see Fig. 1 and 2 of Weber \& Davis 1967, solution $\left.u_{\alpha 1}\right)$. Therefore, we shaped our approach in such a way that we automatically select solutions having this topology.

Each solution is characterised by the 10 parameters described in Table (1). In particular, $F$ and $\Gamma$ describe the magnetic field configuration and the kind of plasma in the system, therefore they can be considered as defined for a given class of outflows. The magnetisation parameter, $\sigma_{\mathrm{M}}$, gives the efficiency with which matter is pulled out of the rotating plasma at the base of the jet. As shown in Table (1), it is a function of the angular velocity of the streamlines, the magnetic field distribution and mass load. Hence, for a jet to be efficiently launched, it has to be strongly magnetised, rapidly rotating and/or have little mass load, as pointed out by Fendt \& Ouyed (2004). The $\sigma_{\mathrm{M}}$ parameter could be used as a fitted parameter, like we did to reproduce PMM14's results, but in general we keep this fixed, assuming again that this parameter will be defined by specific applications. Following a similar logic, we usually fix the position and the inclination of the field line, and the radial distance from the axis of the Alfvén point with $\theta_{\mathrm{A}}, \psi_{\mathrm{A}}$ and $\varpi_{\mathrm{A}}$. This last parameter is related to the strength of the gravitational potential as described in Appendix A. We will discuss in detail the role of the fixed parameters in Section 5. In principle, the rest of the parameters should follow from the integration of the system of equations (1)-(4) once a suitable set of initial conditions is given for $M^{2}, G^{2}$ and $\theta$ (see VK03).

The integration is not simple, however, because the ini- 
tial location of the MSP and MFP is not known. Therefore, some of the unknown parameters must be fixed, while the remaining ones are being retrieved as part of the solution process. The constraint we have on the solution, i.e. that all three singular points must be crossed, determines how many parameters will be found and how many need to be fixed a priori. The procedure that we follow is then an iterative one: 1) we fix a set of parameters $\left.\left(F, \Gamma, \sigma_{\mathrm{M}}, \varpi_{\mathrm{A}}, \theta_{\mathrm{A}} \psi_{\mathrm{A}}\right), 2\right)$ we make an educated guess for the remaining ones $\left(\theta_{\mathrm{MSP}}\right.$, $\left.\left.\theta_{\mathrm{MFP}}, q, x_{\mathrm{A}}^{2}\right), 3\right)$ we derive initial conditions for the integration and integrate the equations, and finally 4) we evaluate the "goodness" $\chi$ of the solution, improve the guesses and integrate again until a high enough $\chi$ is achieved. We now justify and describe in more detail our approach for each of these steps.

\subsection{Fixed and guessed parameters}

As a basic requirement we impose that all our solutions cross smoothly all the three critical points: MSP, AP, MFP (see Fig. 2). At the AP analytical conditions are known (see VK00). Given all the parameters ${ }^{3}$, these conditions allow the determination of $G_{\mathrm{A}}^{2}, M_{\mathrm{A}}^{2}, d G^{2} /\left.d \theta\right|_{\mathrm{A}}$, and $d M^{2} /\left.d \theta\right|_{\mathrm{A}}$ through the Alfvén regularity condition (ARC, see Appendix A), thus allowing integration away from the AP.

The regularity conditions at the modified magnetosonic points MFP and MSP are not known analytically. They are both of the same kind: the denominator $\mathcal{D}$ and the the numerators $\mathcal{N}_{1}$ and $\mathcal{N}_{2}$ of Eqs. (1) and (5) must vanish, while $d M^{2} / d \theta$ and $d \psi / d \theta$ remain finite. Other authors, like VK00, VK03, PMM13, PMM14, found their solutions by using a shooting method to integrate from the Alfvén point upstream towards the MSP and downstream towards the MFP. This method has major caveats, however, since it is very hard to numerically integrate towards singular points, in which the numerators and the denominator approach zero simultaneously, while keeping the accuracy of the solution. It is however numerically stable to integrate away from a singularity. This inspired us to explore a different approach.

We first guess the locations of the critical points, $\theta_{\mathrm{MFP}}$ and $\theta_{\mathrm{MSP}}$ and derive values for $M^{2}, G^{2}$ and their derivatives with respect to $\theta$ based on the regularity conditions $\mathcal{N}_{1}=\mathcal{N}_{2}=\mathcal{D}=0$ evaluated at the $\theta_{\mathrm{MFP} / \mathrm{MSP}}$ of choice (see Appendix $\mathrm{C}$ for our numerical technique). We then are able to integrate away from each initial guess for the modified magnetosonic points and avoid numerical inaccuracies. At the same time, we integrate away from the AP towards both magnetosonic points and consider how good the match is for the values of $M^{2}$ and $G^{2}$ of the various solutions at the midpoints $\theta_{\text {mid, }}$ MFP $=\left(\theta_{\mathrm{A}}+\theta_{\mathrm{MFP}}\right) / 2$ and $\theta_{\text {mid, MSP }}=\left(\theta_{\mathrm{A}}+\theta_{\mathrm{MSP}}\right) / 2$ (see Fig. 3). These are in total four conditions, which imply four free parameters. $\theta_{\mathrm{MFP}}$ and $\theta_{\mathrm{MSP}}$ are two necessary ones and we are left with the freedom to choose two other parameters. We chose to leave $x_{\mathrm{A}}^{2}$ and $q$ free and fix the others. This choice is the most natural and convenient one: $x_{\mathrm{A}}^{2}$ immediately determines $M_{\mathrm{A}}^{2}$ as per Eq. A15, while knowing $q$ and the position of the AP allows our algorithm to derive $d M^{2} /\left.d \theta\right|_{\mathrm{A}}$ very quickly.

\footnotetext{
${ }^{3} \theta_{\mathrm{A}}, \varpi_{\mathrm{A}}$ and $x_{\mathrm{A}}^{2}$ determine the position of the AP, but $F, \Gamma, q$, $\sigma_{\mathrm{M}}$ and $\psi_{\mathrm{A}}$ are needed for $M^{2}$ and $G^{2}$ and their derivatives.
}

\subsection{Initial conditions at the singular points}

As mentioned above, the AP is completely determined. As for the two modified magnetosonic points, there is no analytical condition that can be used to regularize the equations. We use a combination of root-finding techniques that allow us to find the values of $M^{2}$ and $G^{2}$ that give $\mathcal{N}_{1}=$ $\mathcal{N}_{2}=\mathcal{D}=0$. A similar procedure can be applied to both the MFP and the MSP. Once the values of $M^{2}$ and $G^{2}$ at the singular points are found, the last step before starting the integration is finding the derivative of $M^{2}$. By making use of Eq. 2 and of $M^{2}$ and $G^{2}$ at the points of interest, we determine $d M^{2} / d \theta$ by finding the root of the function C5. The integration from all the three singular points can now start. This step is very important, serving as a numerical regularity condition, but it is somewhat laborious, so that for a more detailed discussion, we refer the interested reader to the Appendix C.

At this stage we have both initial values and derivatives for $M^{2}$ and $G^{2}$, evaluated at $\theta_{\mathrm{MFP} / \mathrm{MSP}}$, and we can finally start the integration inwards towards the AP. Since we integrate away from singular points, a standard adaptive step Runge-Kutta scheme is sufficient to integrate Eqs. (1) and (2) (giving $M^{2}$ and $G^{2}$ ), while we retrieve $\psi$ from Eq. (3) and $\xi$ from Eq. (4). Thanks to this procedure it is also possible to integrate downstream from the MFP further away from the black hole and upstream from the MSP towards the equatorial plane of the disk: we simply repeat the same procedure on the other side of the critical points.

\subsection{Goodness of solution and solution finding}

In order to find the best parameters $\left(\theta_{\mathrm{MSP}}, \theta_{\mathrm{MFP}}, q, x_{\mathrm{A}}^{2}\right)$ for a given problem set $\left(F, \Gamma, \sigma_{\mathrm{M}}, \varpi_{\mathrm{A}}, \theta_{\mathrm{A}} \psi_{\mathrm{A}}\right)$ which minimise the offsets at each midpoint simultaneously (see Fig. 3), we make use of the open-source Bayesian inference algorithm multinest (Feroz \& Hobson 2008; Feroz et al. 2009, 2013). multinest is a very robust software package which is also fast due to MPI parallelisation. It also has the advantage of not requiring derivatives of the fitted function, like for example the Newton-Raphson method. This makes it even better suited for our case, because the derivatives can only be calculated numerically and such an approach would increase the numerical error and would prevent most attempts at finding a good fit. We only use the fitting algorithm of multinest. In this case, the only information that needs to be given to the algorithm is the goodness of fit of a given calculation. It then proceeds to maximise such a function by exploring the given domain of the free parameters ${ }^{4}$.

To quantify the goodness of fit of a single integration, we measure the mismatch at the offsets between the values of $G^{2}$ and $M^{2}$ from the integration from AP and the modified magnetosonic points, summing the relative differences of all variables:

$$
\chi=\left[f_{\mathrm{G}, \mathrm{F}}^{2}+f_{\mathrm{M}, \mathrm{F}}^{2}+f_{\mathrm{G}, \mathrm{S}}^{2}+f_{\mathrm{M}, \mathrm{S}}^{2}\right]^{-1 / 2}
$$

\footnotetext{
4 We set a flat prior for all parameters.
} 

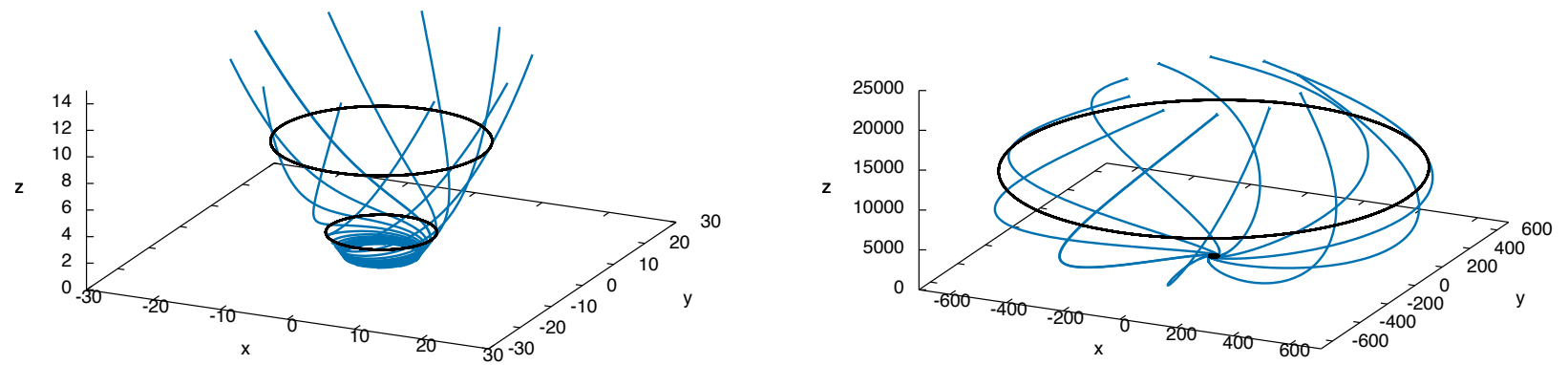

Figure 2. 3D plot of the streamlines corresponding to model (6) in Tab. 2 (see Fig.1a in Contopoulos (1995) for a comparison). Left: Zoom at the base (MSP and AP black circles, starting from the bottom respectively). Right: MFPs.

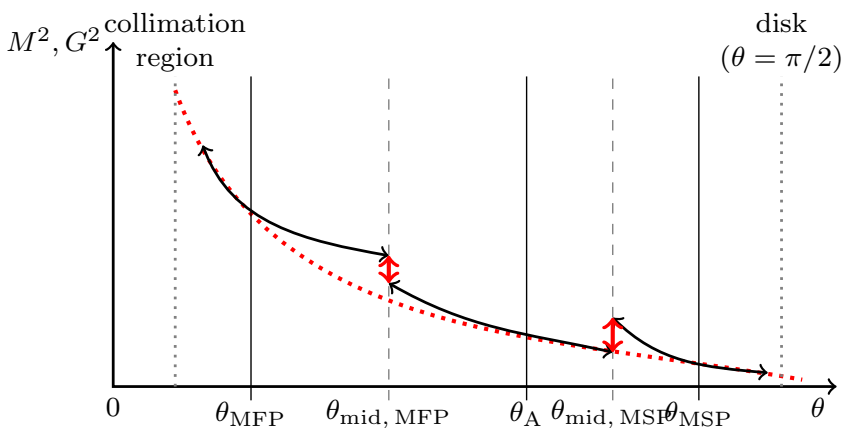

Figure 3. Schematic of the method to derive the form of the functions $M^{2}(\theta)$ or $G^{2}(\theta)$. The solid black lines are the branches of integration with the corresponding direction shown with arrows. A typical situation where the given set of input parameters plus the guesses for the $\theta_{\mathrm{MFP}}$ and $\theta_{\mathrm{MSP}}$ and $x_{\mathrm{A}}^{2}$ and $q$ at these points do not provide a smooth solution through all the singular points and we see large offsets at the midpoints (red solid arrowed lines), while the closest good solution looks like the red dotted line. Note that the last integration branches towards the disk and downstream of MFP are not used in the evaluation of the fitness, but calculated at later times.

where

$$
f_{\mathrm{G}, *}=\left.\frac{2\left(G_{L}^{2}-G_{R}^{2}\right)}{\left(G_{L}^{2}+G_{R}^{2}\right)}\right|_{\theta_{\mathrm{mid}, *}}, \quad f_{\mathrm{M}, *}=\left.\frac{2\left(M_{L}^{2}-M_{R}^{2}\right)}{\left(M_{L}^{2}+M_{R}^{2}\right)}\right|_{\theta_{\mathrm{mid}, *}}
$$

and the subscripts $L$ and $R$ stand for left and right, such that at $\theta_{\text {mid, MFP }} L$ is the result of the integration from the MFP and $R$ the results of the integration from AP; while

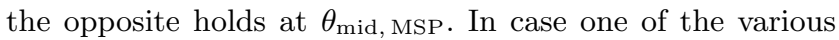
preparatory steps described in Appendix C fails, we are unable to calculate $\chi$ and simply return zero, discarding the corresponding point in the parameter space. We accept a solution and stop the iterations when we reach a fitness value ${ }^{5}$

${ }^{5} \chi$ is the inverse RMS of all the fitting errors. Double precision in a computer gives up to $\sim 10^{-14}-10^{-16}$ machine roundoff error, but roundoff errors can build up in any calculation. So, if the chosen $1 / \chi$ is too small, convergence could suffer. We found $10^{-9}$ to be a good compromise choice. of $\chi \geq 10^{9}$. During the process of finding the solutions we noted that there is a highly non-linear relation between the amount of change of the different parameters $q, x_{\mathrm{A}}^{2}, \theta_{\mathrm{MFP}}$ and $\theta_{\mathrm{MSP}}$ and the resulting change in the fitness function Eq. (12), such that each parameter should be known with at least six significant digits to make a good fit.

\section{COMPARISON WITH PREVIOUS SOLUTIONS}

We start our parameter study by recovering the solutions presented in Table 1 of PMM14. We list our parameters corresponding to PMM14's reference and first solutions in Tab. 2, as models $I$ and $I I$. We identify two factors that can explain the discrepancies in the parameters: 1) differences in the numerical scheme and 2) definition of the functions of the gravitational potential in the gravity terms (Eq. A13 and Appendix B). Comparing then the parameters published in PMM14 (first solution) with model IIa, both listed in Tab.2, we noticed that whilst there is not an appreciable difference in $q$ and $\sigma_{\mathrm{M}}$, which are the fitted parameters for PMM14, the locations of both MFP and MSP change by a few \%.

As discussed in Section 2, if we remove the approximation of a small potential in the gravity terms, we find substantial differences in our solution parameters (see models $I a-I b, I d-I e$ and $I I a-I I b)$, that are clearly seen when drawing the corresponding streamlines: in Fig. 4 we plot the projected streamlines in the $z \varpi$-plane for the models $I a$ to If. Model $I a$ is our reference solution, which differs from PMM14 only for the numerical method used to solve the equations (1)-(4) (black solid line). Model $I b$ is the reference solution with the corrected potential functions (purple dashed line) and model $I c$ is the reference solution with the corrected potential functions, but with Newton potential (green dotted line). It is evident that introducing the approximation of small $P_{\mathrm{g}}$ in the derivatives of $P_{\mathrm{g}}$ itself (see discussion in Sec. 2 and Appendix B) reduces the effect of gravity close to the $\mathrm{BH}$ (right panel in Fig. 4). Larger discrepancies at MSP appear when we consider a relativistic gas with adiabatic index 4/3 (see models Id (blue dot-dashed line), Ie (orange dot-dot-dashed line) and If (yellow long-dashed line)). Generally, the effect of a small Paczyńsky-Wiita po- 
Table 2. Parameter study of the solutions recovered from PMM14. The classes of models $I$ and $I I$ are the "reference" and "first" solutions in Tab.1 of PMM14. Sub-classes of models differ for the adiabatic index $\Gamma$, the gravitational potential, $P_{\mathrm{g}}$ (Newtonian=N or Paczyńsky-Wiita $=\mathrm{PW}$ ), and whether the functions $f_{i}\left(P_{\mathrm{g}}\right)$ are approximated $(\mathrm{A})$ or corrected $(\mathrm{C})$ (see Appendixes A and B). The fitted parameters are shown in italic and with 6 significant digits.

\begin{tabular}{|c|c|c|c|c|c|c|c|c|c|c|c|c|}
\hline Model & $x_{\mathrm{A}}^{2}$ & $q$ & $\theta_{M F P}$ & $\theta_{M S P}$ & $\theta_{A}$ & $\psi_{A}$ & $\sigma_{M}$ & $\varpi_{A}$ & $\Gamma$ & $\mathrm{F}$ & Style & $P_{g}$ \\
\hline Reference & 0.145330 & $2.4184 E-2$ & 0.118635 & 1.26022 & 60 & 40 & 0.02 & 15 & $5 / 3$ & 0.75 & A & PW \\
\hline$I a$ & 0.145329 & $2.41844 E-2$ & 0.118608 & 1.26383 & 60 & 40 & 0.02 & 15 & $5 / 3$ & 0.75 & A & PW \\
\hline$I b$ & 0.158777 & $2.74016 E-2$ & 0.116215 & 1.19411 & 60 & 40 & 0.02 & 15 & $5 / 3$ & 0.75 & $\mathrm{C}$ & PW \\
\hline Ic & 0.143936 & 1.86592E-2 & 0.118492 & 1.31034 & 60 & 40 & 0.02 & 15 & $5 / 3$ & 0.75 & $\mathrm{C}$ & $\mathrm{N}$ \\
\hline$I d$ & 0.228007 & $1.19385 E-3$ & 0.104885 & 1.16340 & 60 & 40 & 0.02 & 15 & $4 / 3$ & 0.75 & A & PW \\
\hline Ie & 0.264180 & $7.43368 E-4$ & 0.104566 & 1.15714 & 60 & 40 & 0.02 & 15 & $4 / 3$ & 0.75 & $\mathrm{C}$ & PW \\
\hline If & 0.225918 & $8.34464 E-4$ & 0.105647 & 1.19239 & 60 & 40 & 0.02 & 15 & $4 / 3$ & 0.75 & $\mathrm{C}$ & $\mathrm{N}$ \\
\hline First solution & 0.01 & $1.4359 E-2$ & 0.120427 & 1.18682 & 60 & 45 & $7.85798 E-4$ & 18.2088 & $5 / 3$ & 0.75 & A & PW \\
\hline$I I a$ & 0.01 & $1.43588 E-2$ & 0.114838 & 1.19846 & 60 & 45 & $7.85794 E-4$ & 18.2088 & $5 / 3$ & 0.75 & A & PW \\
\hline$I I b$ & 0.01 & $1.66386 E-2$ & 0.115231 & 1.12314 & 60 & 45 & $5.94572 E-4$ & 18.2088 & $5 / 3$ & 0.75 & $\mathrm{C}$ & PW \\
\hline$I I c$ & 0.01 & $1.25403 E-2$ & 0.119471 & 1.20381 & 60 & 45 & $7.86151 E-4$ & 18.2088 & $5 / 3$ & 0.75 & $\mathrm{C}$ & $\mathrm{N}$ \\
\hline \multicolumn{13}{|l|}{ Others } \\
\hline (1) & 0.643674 & $6.13069 E-3$ & $8.06108 E-2$ & 1.32117 & 60 & 45 & 0.50 & 15 & $4 / 3$ & 0.75 & $\mathrm{C}$ & PW \\
\hline (2) & 0.324834 & $6.91188 E-3$ & 8.37261E-2 & 1.28111 & 60 & 46 & 0.10 & 15 & $4 / 3$ & 0.75 & $\mathrm{C}$ & PW \\
\hline (3) & 0.258726 & $4.90328 E-3$ & $9.73984 E-2$ & 1.22659 & 60 & 44 & 0.05 & 15 & $4 / 3$ & 0.75 & $\mathrm{C}$ & PW \\
\hline$(4)$ & 0.475936 & $7.87961 E-3$ & $9.30176 E-2$ & 1.27947 & 60 & 44 & 0.20 & 15 & $4 / 3$ & 0.75 & $\mathrm{C}$ & PW \\
\hline (5) & 0.816325 & $1.70893 E-5$ & 7.02122E-2 & 1.27569 & 60 & 44 & 0.75 & 15 & $4 / 3$ & 0.75 & $\mathrm{C}$ & PW \\
\hline (6) & 0.864334 & 6.86982E-6 & $6.27254 E-2$ & 1.33771 & 60 & 47 & 1.45 & 15 & $4 / 3$ & 0.75 & $\mathrm{C}$ & PW \\
\hline (7) & 0.503771 & 2.85668 & $2.58765 E-2$ & 1.38088 & 57.5 & 47 & 1.45 & 15 & $4 / 3$ & 0.85 & $\mathrm{C}$ & PW \\
\hline (8) & 0.470045 & $2.53731 E-5$ & 0.10165 & 1.15853 & 60 & 39 & 0.05 & 15 & $4 / 3$ & 0.75 & $\mathrm{C}$ & PW \\
\hline
\end{tabular}

tential is very similar to a Newtonian potential. We define the last recollimation point (LRP) as the last point of the streamline where the integration downstream of MFP stops. In the left panel of Fig. 4, we note that the positions of the MFP and the LRP lie far apart from each other when comparing solutions with corrected or approximated functions of the gravitational potential.

\subsection{The self-similarity assumption}

As pointed out by PMM14, the self-similarity assumption is a serious limitation intrinsic in the derivation of the equations. The inclusion of gravity further complicates the matter. Radial self-similarity, even without the inclusion of gravity, introduces a few geometrical constraints. Ultimately, to properly quantify these issues, our solutions will be benchmarked against GRMHD simulations in future works. In this section, we focus on determining the effect of gravity on the self-similarity assumption. In Fig. 5 we show two families of solutions obtained varying $\varpi_{\mathrm{A}}$ from the reference models $I a$ using eq. A14 and $I b$ (Tab. 2) using eq. A13. All the solutions found with the the corrected functions of the gravitational potential (eq. A13) can be integrated down to the disk midplane $(z=0)$. Each field line will have a characteristic angular velocity, $\Omega(\varpi, z)$, which is a constant of motion along the field line, but it will vary from one field line to an- other (see Appendix D). As expected from relativistic selfsimilar models, e.g. Li et al. (1992) and VK03, $\Omega(\varpi, z=0)$ follows a profile which is $\propto R^{-1}$. However the gravity terms add some perturbation in the proximity of the black hole. The maximum deviation between the angular velocity of a single field line at $z=0$ compared to $\Omega_{\mathrm{K}}=\left(R / R_{\mathrm{g}}\right)^{-3 / 2}$ $(G=c=M=1)$ is a factor of 2 , with our field lines rotating at lower speed than the keplerian one. All streamlines should look as scaled copies of the same shape; however, where gravity is strong enough, for example upstream of the MSP, the field lines bend and cross each others until $\varpi_{A}$ is larger than $\sim 19 r_{\mathrm{g}}$ (panel $(d)$ in Fig. 5). The MFP is heavily affected as well for smaller values of $\varpi_{\mathrm{A}}(\lesssim 19)$. It is worth noting that for the family of solutions with model $I b$ as reference, we could not obtain solutions for $\varpi_{A}<9$ due to the stronger gravitational potential, while for solutions found around model $I a$, we were able to go down to $\varpi_{A}=5$.

The degree in which self-similarity is affected by gravity depends on the other parameters, such as $\sigma_{\mathrm{M}}$ and $\psi_{\mathrm{A}}$ : we show this in Fig. 6. The four panels in the figure show the values of the fitted parameters of various families of solutions, as a function of $\varpi_{\mathrm{A}}$. In the absence of gravity, self-similarity would ensure that $x_{\mathrm{A}}^{2}, q, \theta_{\mathrm{MFP}}$ and $\theta_{\mathrm{MSP}}$ be constant within the same family of solutions, independent of any variation in the value of the cylindrical radius $\varpi_{A}$. In 



Figure 4. Comparison of stream lines of models Ia to If from Tab. 2. Colours correspond to same model in both panels: black solid line is model $I a$, purple short-dashed line is model $I b$, dotted-green line is model $I c$, dot-dashed blue line is model $I d$, orange dot-dot-dashed line is model $I e$, and yellow long-dashed line is model If. The models $I a, I b$, and $I c$ have adiabatic index of $5 / 3$ and differ for the potential, $P_{\mathrm{g}}$, used (N or PW) and whether the functions $f_{i}\left(P_{\mathrm{g}}\right)$ are approximated $(\mathrm{A})$ or corrected $(\mathrm{C})$. The models $I d$, Ie, and If are the same but with $\Gamma=4 / 3$. Left: MFP region, where the black dots mark the height of the MFP for the corresponding streamline. Right: Zoom on the AP and MSP. The AP (red dot) is fixed in all the solutions, therefore the streamlines converge to the same point with the same derivative. The black dots define the location of the MSP of each streamlines.

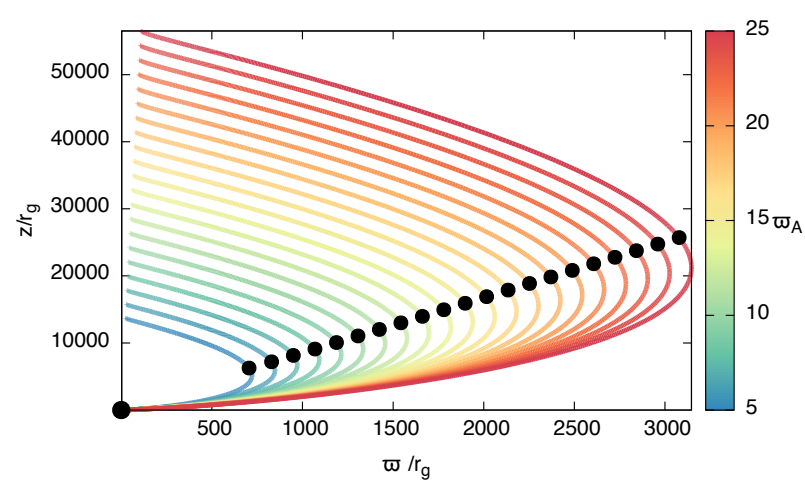

(a)

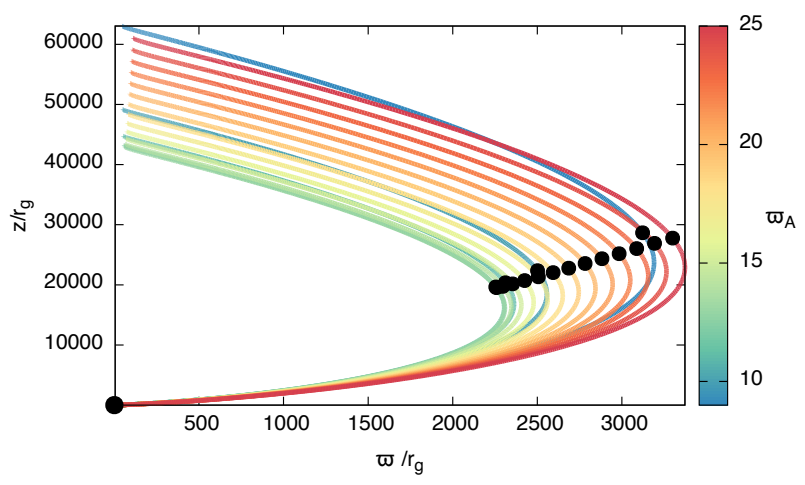

(c)

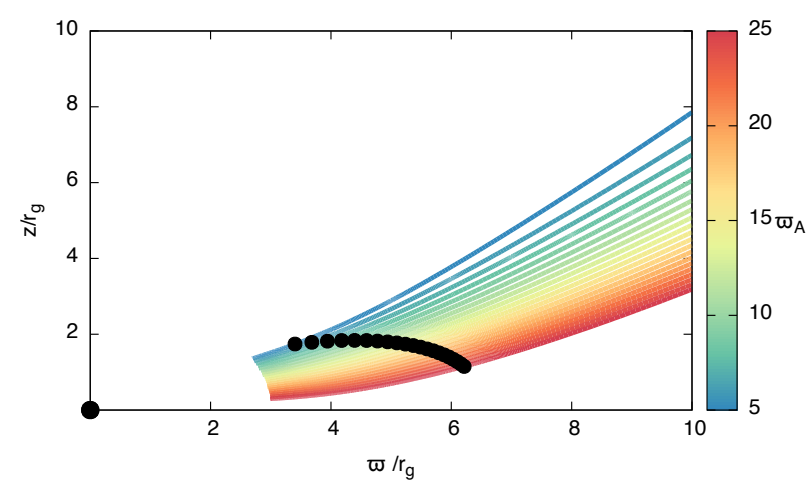

(b)

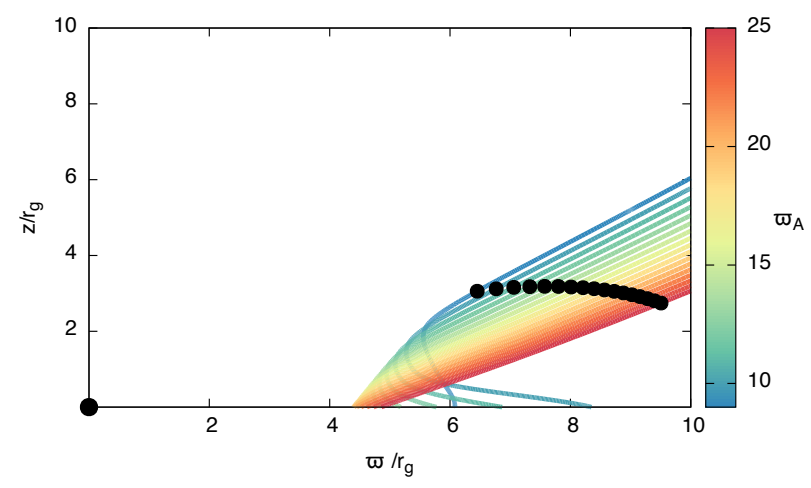

(d)

Figure 5. Family of solutions obtained by varying the cylindrical radius of the Alfvén point, $\varpi_{A}$. The reference solution $\left(\varpi_{A}=15\right)$ for the upper panels is model $I a$ and for the bottom panels is $I b$ in Tab. 2. Upper panels: We vary $\varpi_{A}$ in the interval $5-25$, as the lines go from blue to red. On the left, the black dots on the curves mark the position of the MFP, while on the right they are showing the MSP. The bigger black dot at the origin of the axis is the BH. Lower panels: Same but the interval is restricted to $9-25$. 
Fig. 6 we show families that correspond to four models of Tab.2: Ia (purple), Ib (green), 1 (light blue), 2 (orange).

Solutions corresponding to model $I a$ and $I b$ have lower $\psi_{\mathrm{A}}\left(=40^{\circ}\right)$ and lower $\sigma_{\mathrm{M}}(=0.02)$ with respect to model (1) and (2) which have $\psi_{\mathrm{A}}=45^{\circ}, \sigma_{\mathrm{M}}=0.5$ and $\psi_{\mathrm{A}}=$ $46^{\circ}, \sigma_{\mathrm{M}}=0.1$, respectively. When we introduce gravity, we are introducing a disturbance in the radial scaling of the streamlines, that becomes more pronounced as we move closer to the BH, i.e. $\varpi_{A} \lesssim 15$. Gravity breaks self-similarity for all the parameters at play, but its effect is more dramatic on $\theta_{\text {MSP }}$. Nonetheless, the median of the absolute deviations from the results' median $\left(\operatorname{mad}=\operatorname{median}\left|X_{i}-\operatorname{median}(X)\right|\right)$ for the parameters $x_{\mathrm{A}}^{2}, q, \theta_{\mathrm{MFP}}, \theta_{\mathrm{MSP}}$ over the full interval of $\varpi_{A}$ is at most $\sim 10 \%$. This gives us the confidence in the use of this method.

\section{PARAMETER SPACE STUDY}

We present here a more detailed analysis of an ensemble of solutions found in a $\left(\psi_{\mathrm{A}}, \sigma_{\mathrm{M}}\right)$ slice of the parameter space, for a specific choice of a subset of fixed parameters, namely $F=0.75, \theta_{\mathrm{A}}=60^{\circ}, \Gamma=4 / 3$ and $\varpi_{\mathrm{A}}=15$. Although we fixed it for all runs, $\varpi_{\mathrm{A}}$ has been changed when testing self-similarity for models $I a, I b,(1)$ and (2) of Tab. 2. Changing $\varpi_{\mathrm{A}}$ changes the radius of the stream line, allowing one to check self-similarity of a given outflow with the parameters $F, \theta_{\mathrm{A}}, \sigma_{\mathrm{M}}$ and $\Gamma$. We populate a two-dimensional grid of solutions by varying $\psi_{\mathrm{A}}$ and $\sigma_{\mathrm{M}}$, while fitting for $x_{\mathrm{A}}^{2}, q, \theta_{\mathrm{MFP}}$ and $\theta_{\mathrm{MSP}}$ and keeping all the other parameters fixed. In Tab. 2, models (3)-(6) belong to this grid. We use them to present features also found in other solutions in the grid. Models (7) and (8) are solutions found in other areas of the parameter space and we include them into the discussion to show their peculiar characteristics. Our primary goal in such exploration is to expand the pool of solutions found by PMM14 and search for a wider range of relativistically boosted jet solutions, i.e. with bulk Lorentz factors in the range of $2-10$, in order to find suitable solutions for future applications to astrophysical objects. Considering the difficulties of starting from a random initial position, we started by changing the polytropic index to $4 / 3$ and keeping the other fixed parameters as in the reference solution in PMM14 (first row in Tab. 2), then slowly increasing $\sigma_{\mathrm{M}}$ and $\psi_{\mathrm{A}}$ until it was possible to find solutions. As we can see from Fig. 7, the parameter space is not a continuous volume and has patches where no solution exists. Due to the high dimensionality of this space, it is often difficult to know $a$ priori where solutions can be found. We will focus on the nature of these boundaries later on in this section.

The solutions found in the $\left(\psi_{\mathrm{A}}, \sigma_{\mathrm{M}}\right)$ slice presented here, lie on a three-dimensional curve which extends on a limited range of the interested parameters. Although solutions with $\sigma_{\mathrm{M}}<0.05$ could be recovered, we dedicated little time to the exploration of this class of solutions since they appear to have very slow bulk velocities, $\gamma_{\mathrm{MFP}} \gtrsim 1$.

Just from the solutions in this sparse 2-dimensional grid, we see already a variety of flow shapes and dynamics. For instance, we show in Fig. 8 six examples of the dynamical evolution of the energy terms (Eq. 6) along the jet streamlines corresponding to the solutions (3) to (8) in Table 2 . The panels $(a)-(c)$ differ in the value of $\sigma_{\mathrm{M}}$, and indeed the Poynting energy at the base increases going from $(a)$ to $(c)$, while the enthalpy and kinetic energy remain unchanged. At the launching site, the jet can be powered by a different source of energy depending on the parameters, being first thermally-dominated $\left(0.05 \geq \sigma_{\mathrm{M}}<0.2\right.$, panel $\left.(a)\right)$, crossing equipartition between magnetic and thermal energy at $\sigma_{\mathrm{M}}=0.2$ (panel $(b)$ ) and later becoming magneticallydominated $\left(0.2<\sigma_{\mathrm{M}} \leq 0.75\right.$, panel $\left.(c)\right)$. However, we note that all these solutions have very little thermal energy overall, so they are still relatively "cold" jets. When $\sigma_{\mathrm{M}}$ increases even further (panel $(d)$ ), the Poynting energy completely overtakes all the other energy contributions until it converts entirely into kinetic energy at $\sim 1000 r_{\mathrm{g}}$. A common feature of all our jet solutions is the conversion of the primary source of energy (enthalpy or Poynting energy) at the base into kinetic energy at some distance from the $\mathrm{BH}$ between the Alfvén point and the MFP, as it is to be expected for relativistic flows (Komissarov et al. 2010). The jet is always kinetically-dominated by the time it approaches the MFP. There is another channel for the energy exchange, however, which we discuss below.

\subsection{Counter-rotation in hot jets}

Panel (e) of Fig. 8 shows the energy components of model (7). This jet is roughly at equipartition at its foot point, with both enthalpy and Poynting energy being large. This is a hot, magnetized jet. We see that, starting from the MSP, the Poynting energy increases as a consequence of the transfer of a fraction of the thermal energy into the magnetic field, while the kinetic energy increases at lower pace. The fraction of energy transferred between the different components is regulated by the conservation of the total energy $\mu^{\prime}$. This third channel of energy transfer from the kinetic and thermal components to the magnetic component has never been seen before in a semi-analytical model, although present in simulations (see model $\mathrm{B} 2 \mathrm{H}$ and Section 5.5 in Komissarov et al. 2009) and discussed analytically by Sauty et al. (2012) and Cayatte et al. (2014). This result is thus important because it demonstrates that our semi-analytical framework produces the full range of flows seen also in MHD simulations, and is not limited to the simplest scenarios. The increase of the Poynting energy also results in an increase in the magnetic component of the angular momentum $L_{\mathrm{M}}^{\prime}$ in Eq. (7) that corresponds to $L_{\mathrm{HD}}^{\prime}$ becoming negative (see right panel Fig. 9 and figure 2 in Cayatte et al. 2014). The change in sign of $L_{\mathrm{HD}}^{\prime}$ is due to $V_{\phi}$ becoming negative (see left panel Fig. 9). In the cold regime, we normally see both components of the velocity, $V_{\mathrm{p}}$ and $V_{\phi}$, always above zero. The jet starts off with a larger toroidal velocity that then decreases in correspondence to the poloidal component taking the lead. In the case of hot magnetized solutions, the toroidal velocity can become negative before the canonical behaviour of a cold jet is restored at larger distances from the black hole. This inversion of sign of the toroidal component of the velocity is interpreted as a counter-rotation of the jet with respect to the disk. Sauty et al. (2012) and Cayatte et al. (2014) claim that the counter-rotation in jets is the signature of the magnetization of the jet and it can be due to several effects: deceleration of the flow, steep gradients of the magnetic field and/or energy transfer from enthalpy to the magnetic field. Model (7) belongs to the latter case. In 


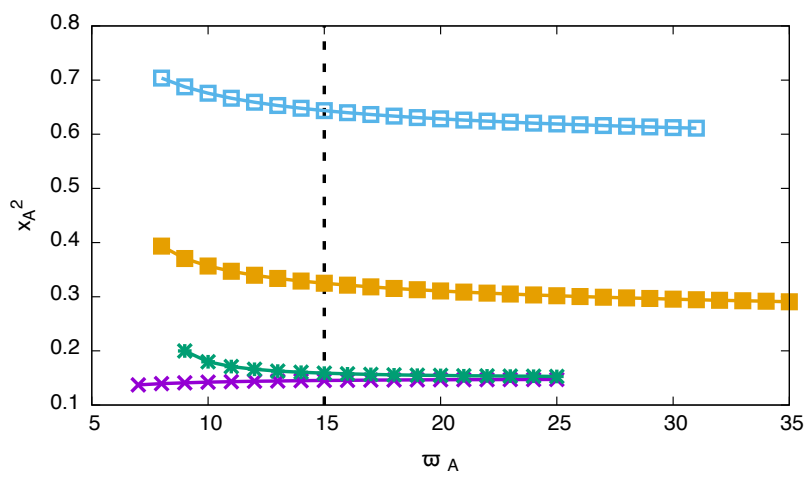

(a)

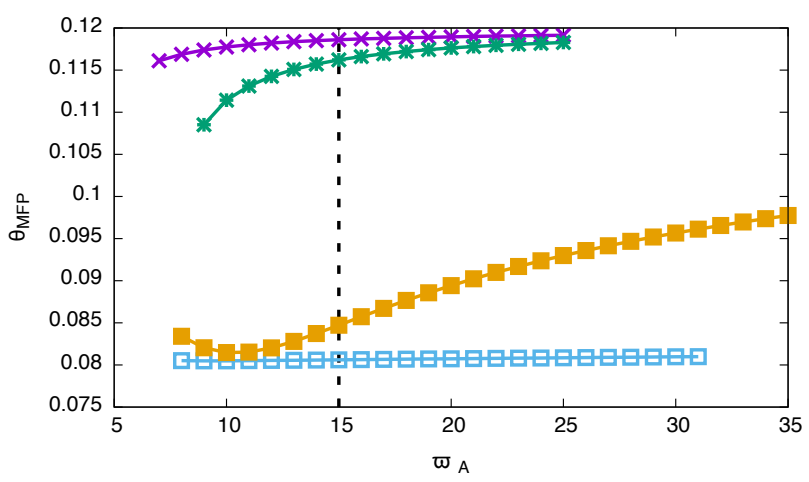

(c)

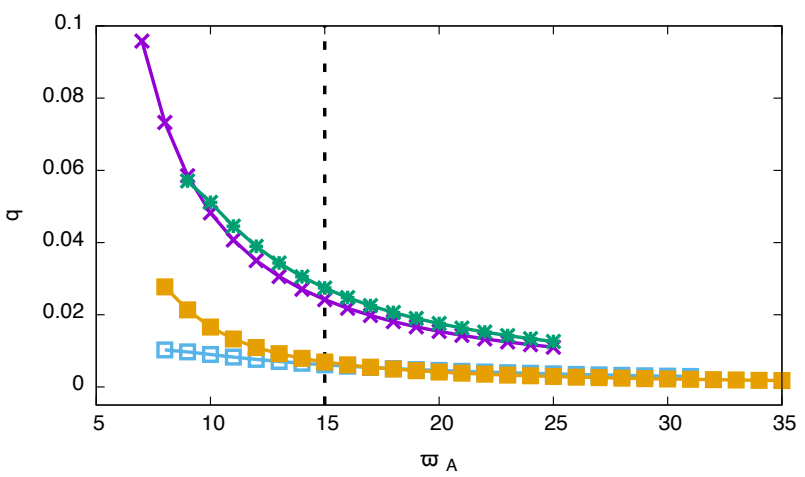

(b)

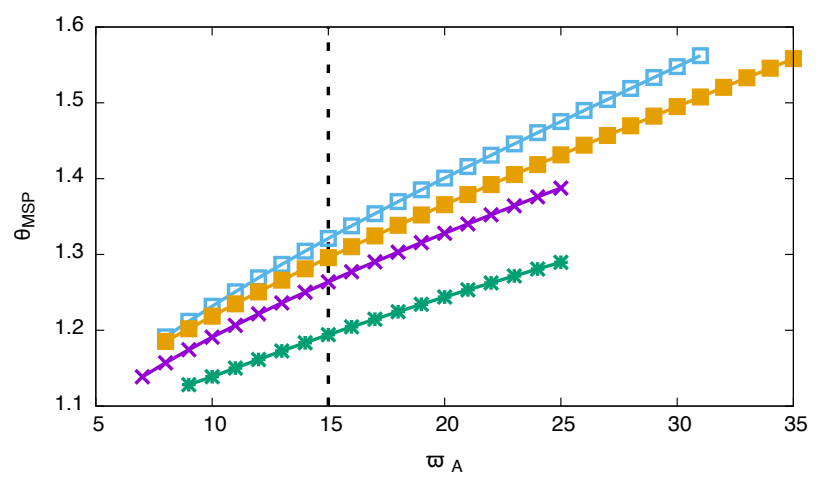

(d)

Figure 6. Fitted parameters $\left(x_{\mathrm{A}}^{2}, q, \theta_{\mathrm{MFP}}, \theta_{\mathrm{MSP}}\right)$ of the same families of solutions as function of the Alfvén cylindrical radius, $\varpi_{A}$. The purple line with crosses and the green line with stars represent the families of solutions corresponding to the reference parameters of the model $I a$ and $I b$ in Tab. 2, respectively. The blue line with hollow squares and the orange line with filled squares are derived from the reference solutions given in Tab. 2 as model (1) and (2) respectively. The vertical dashed lines mark the reference $\varpi_{A}$.
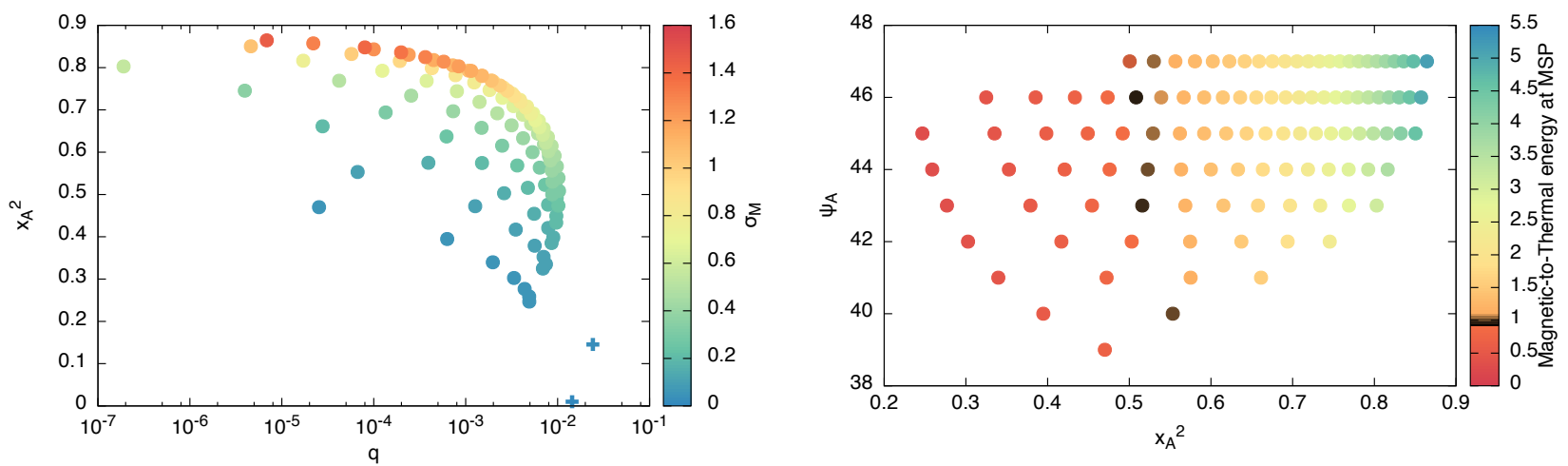

Figure 7. Two-dimensional plots $\left(x_{\mathrm{A}}^{2}\right.$ versus $q$ in the left panel and $\psi_{\mathrm{A}}$ versus $\left.x_{\mathrm{A}}^{2}\right)$ of the parameter space we explored by making discrete steps in the parameters $\psi_{\mathrm{A}}$ and $\sigma_{\mathrm{M}}$, while fitting for $x_{\mathrm{A}}^{2}, q, \theta_{\mathrm{MFP}}$ and $\theta_{\mathrm{MSP}}$. The third dimension $\left(\sigma_{\mathrm{M}}\right.$ in the left panel and $S / \xi \gamma$ with $S=-\varpi \Omega B_{\phi} / \Psi c^{2}$ at the MSP in the right panel) is expressed with the colour scheme on the right of each panel. The two crosses in the left panel are the first and reference solutions of PMM14 with corrected gravity terms. In the right panel, we show which solutions of the grid are magnetically or thermally dominated at the base. The black dots are the solution that have roughly equipartition between magnetic and thermal energy at the base. On the left of these all solutions are thermally powered jets at the base, while on the right there are solutions that are Poynting dominated at the base.. 
Fig. 9 we start from model (7) and progressively decrease F. In the left panel, we see how for higher $F$ the solutions become hotter and their toroidal velocity minima decrease and they exhibit counter-rotation.

Similar interplay between energy terms can also result in almost oscillatory behaviours in the evolution of the energy components, seen for instance when the initial amount of enthalpy in the system is close to its minimum $(=1$, i.e. $q \sim 10^{-5}-10^{-9}$ ) and $\sigma_{\mathrm{M}}<1$ (panel $(f)$ of Fig. 8, depicting solution (8) of Tab. 2). Here we see two episodes where the energy is transferred to the magnetic field from the thermal energy and the flow is decelerating. However, the toroidal component of the velocity does not reverse sign, therefore no counter-rotation is established. This behaviour is usually associated to a highly wound-up streamline below MSP. The radial distance of the streamline during this phase is not constant, inducing a small change in the gravitational potential. Usually, after an initial acceleration powered by the leading force, either thermal or magnetic, the flow starts decelerating while approaching the jet axis, then the gravitational potential becomes relevant again, leading to an acceleration of the flow. Such interplay of forces can happen a few times and it results in complete winding up of the field line before the jet gains enough poloidal velocity to be launched outwards after crossing MSP. If both $q$ and $\sigma_{\mathrm{M}}$ are small, upstream of the MSP the jet has little poloidal component in its velocity, therefore it keeps slowly spiralling upwards, until it gains enough poloidal velocity to be slung out of the disk. Solutions with lower $\psi_{\mathrm{A}}$ could not be found because the jet will not have enough initial energy to acquire sufficient poloidal velocity to then be launched out.

\subsection{On the effect of $\sigma_{\mathrm{M}}$ and $\psi_{\mathrm{A}}$}

We show two examples of the evolution of the toroidal and poloidal components of the velocity for two sets of solutions in Fig. 10, obtained by varying $\sigma_{\mathrm{M}}$ and $\psi_{\mathrm{A}}$. We note that at small $z$ (upstream of the MSP) as $\sigma_{\mathrm{M}}$ increases (left panel of Fig. 10) the toroidal component of the velocity increases, while the poloidal component first becomes larger and then it starts decreasing. For increasing $z$ the toroidal component decreases towards the MFP, but, as $\sigma_{\mathrm{M}}$ increases, a maximum appears around the MSP. The poloidal velocity increases with distance from the black hole and accelerates, rapidly approaching the speed of light close to the MFP/LRP. Indeed, we cannot find solutions with a larger magnetisation parameter for the given set of input parameters $\left(\psi_{\mathrm{A}}=46^{\circ}, \theta_{\mathrm{A}}=60^{\circ}, F=0.75, \Gamma=4 / 3\right.$ and $\left.\varpi_{\mathrm{A}}=15\right)$. In the right panel of Fig. 10, we show a similar plot of the velocity components for a series of solutions obtained by increasing $\psi_{\mathrm{A}}$ and fixing $\sigma_{\mathrm{M}}=0.55, \theta_{\mathrm{A}}=60^{\circ}, F=0.75, \Gamma=$ $4 / 3$ and $\varpi_{\mathrm{A}}=15$. We see that varying either $\psi_{\mathrm{A}}$ or $\theta_{\mathrm{A}}$ individually restricts the search to a small range in such parameters. From the ARC (See Appendix A, Eq. A20), we can put a constraint on the sum of these two angles in order to select solutions that have a negative derivative of the poloidal Mach number, $d M^{2} / d \theta$ (Eq. 1), at the Alfvén point $\left(90^{\circ}<\theta_{\mathrm{A}}+\psi_{\mathrm{A}}<180^{\circ}\right)$. This interval for the sum of $\theta_{\mathrm{A}}$ and $\psi_{\mathrm{A}}$ ensures that the flow is accelerating while moving away from the black hole. From the evolution of the velocity components with respect to this sum we see that the range where we can find solutions, for a given set of fixed parameters, is much smaller than the one inferred from the sign of $d M^{2} / d \theta$ at Alfvén and that the solution can be radically different between the lower end and the upper end of the range. We also note that the larger $\psi_{\mathrm{A}}$ becomes, the closer the MFP moves towards the black hole. We cannot find solutions for $\psi_{\mathrm{A}}>47^{\circ}$ probably because the LRP happens before the MFP, while our method is focused on finding solutions that pass through all the three singular points. When $\psi_{\mathrm{A}}$ decreases, we see that suddenly the jet has a much larger toroidal velocity, while the poloidal component is almost zero. The last solution is therefore the one for which the jet can still be launched from beyond MSP, while it keeps circulating upstream of this point.

\subsection{Exploring other regions of the parameter space}

We already presented here a few solutions that were found outside the initial 2-dimensional grid found by varying $\psi_{\mathrm{A}}$ and $\sigma_{\mathrm{M}}$. Driven by the need to better understand which parameters lead to a shift in the observable values, such as the Lorentz factor of the jet at the MFP/collimation region, the height of the MFP, the energy balance at the launching site, we chose to try to explore different regions of the parameter space, i.e. varying previously fixed parameters. In the left panel of Fig. 11 we show some of the alternative directions that we pursued. We note that each of the parameters used has a substantial effect in determining the height of the MFP compared to the relatively small steps we adopted in this search. Also, the variation of some of them (particularly $\sigma_{\mathrm{M}}$ ) result in large changes in the bulk Lorentz factor of the jet at MFP, e.g. a $\Delta \sigma_{\mathrm{M}} \sim 1.2$ around $\sigma_{\mathrm{M}}=0.70$ corresponds to $\Delta \gamma_{\mathrm{MFP}} \sim 4$.

We find that increasing $F$, thereby changing the scaling of the magnetic field $\left(B \propto r^{F-2}\right)$, is the most efficient way of moving into a different area of the parameter space, letting us touch terminal Lorentz factors of about 11. However, as discussed e.g. in Blandford \& Payne (1982), Contopoulos (1995) and VK03, the higher $F$ the more the MFP is moving to larger distances from the $\mathrm{BH}$, eventually to infinity. Although in principle there is no constraint in our algorithm, with the exception of loss of numerical accuracy, on how high the MFP can lie with respect to the $\mathrm{BH}$, we indeed have not yet found solutions with $F \gtrsim 0.9$ for the parameters explored so far due to the aforementioned numerical accuracy issues, therefore all our solutions are in the so-called return-current regime, i.e. the current decreases with radius.

As a further general feature shared by all solutions, we point out that they are suddenly terminated soon after the MFP, while rapidly recollimating towards the jet axis (see also Fig. 2). From a numerical point of view that happens because the denominator of the wind equation (Eq. 1) and the equation for $\psi$ (Eq. 5 ) goes to zero, $D \rightarrow 0$. We see that the streamlines are rapidly becoming vertical $(\psi \rightarrow \pi / 2)$. At the moment it is difficult to say whether this is due to physical effects such as compression of the gas or just due to the "polar axis singularity" imposed by the self-similarity assumption, which makes the equations degenerate for $\theta \rightarrow$ 0 . 


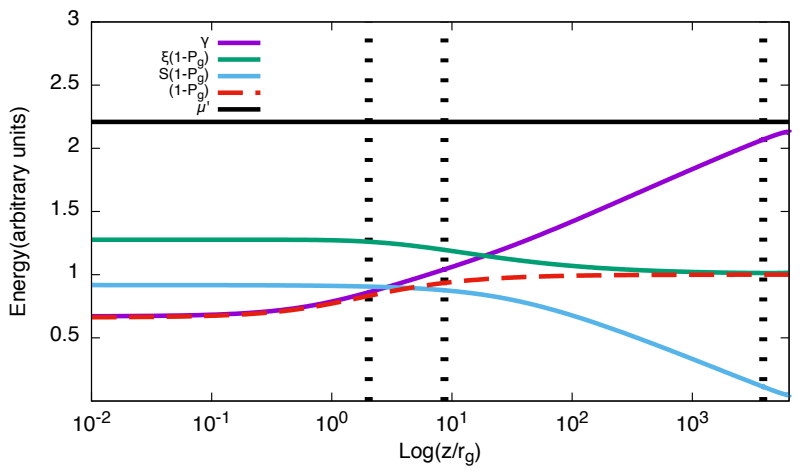

(a)

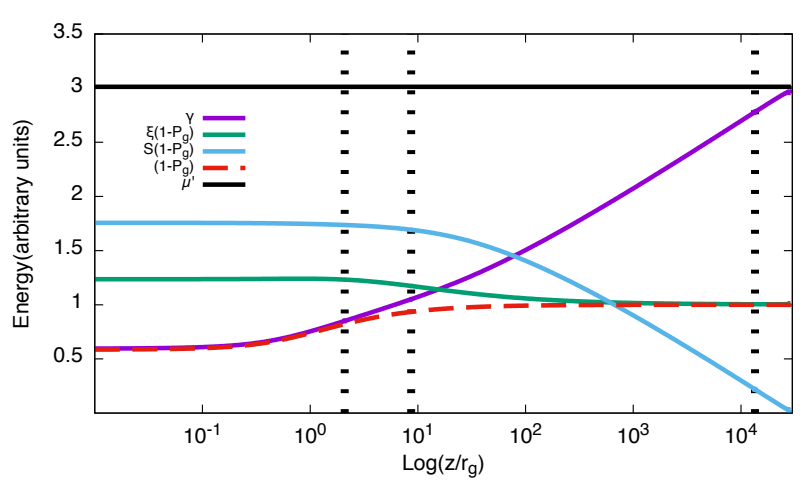

(c)

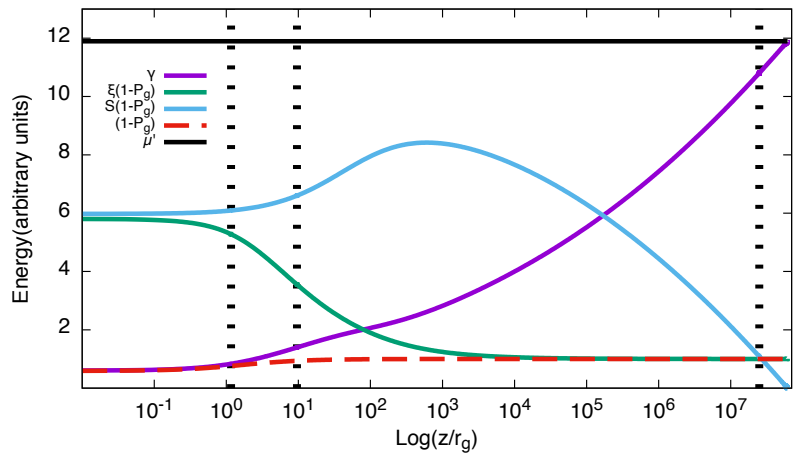

(e)

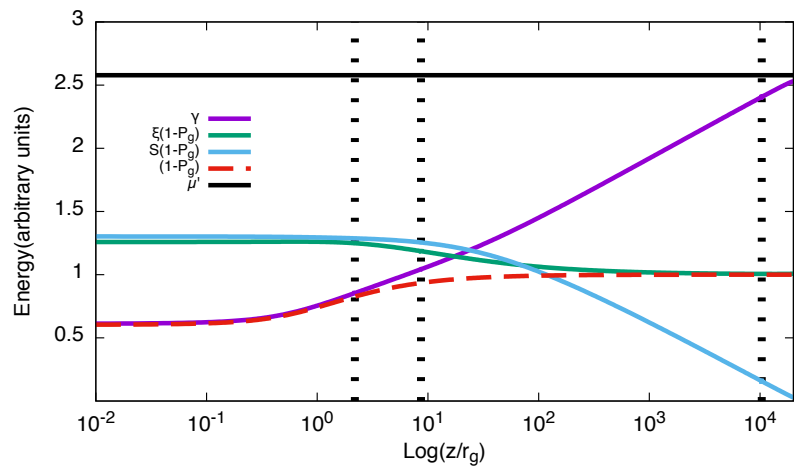

(b)

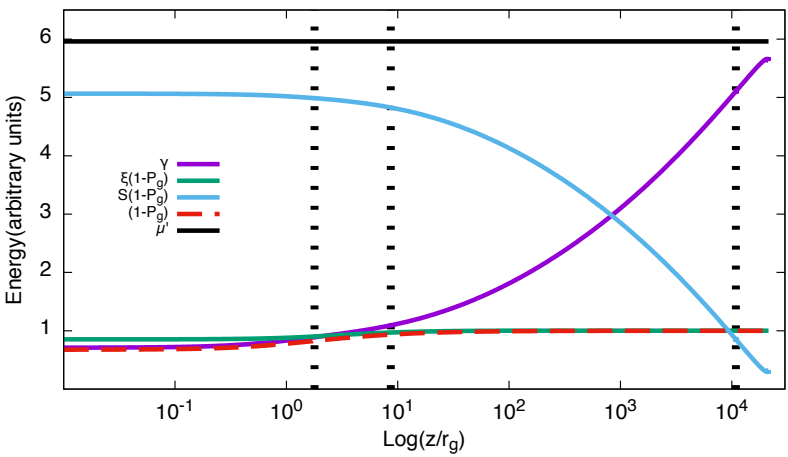

(d)

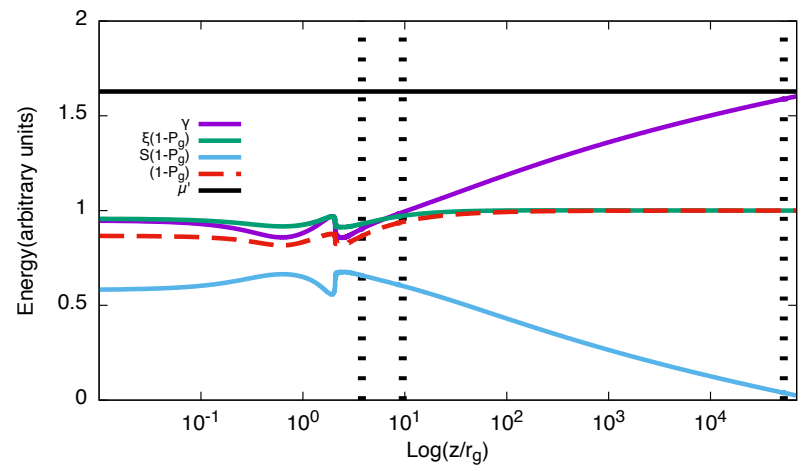

(f)

Figure 8. Evolution of the energy components along the jet streamlines. The black dotted lines indicate the position of the three singular points (from left to right, MSP, AP and MFP). The black solid line is the total energy, $\mu^{\prime}$, the green line is the specific enthalpy, $\xi \gamma$, the light blue line is the energy carried by the magnetic field $-\varpi \Omega B_{\phi} / \Psi$, the purple line is the kinetic energy $\gamma$ and the dashed red line is the function $\left(1-P_{\mathrm{g}}\right)$ of the gravitational potential. As described in Section 2 (Eq. 6), each component is multiplied by this function to account for gravity in the energy balance. Panel $(a)$ shows the energy balance for model $(3)$ with $\sigma_{\mathrm{M}}=0.05, F=0.75$, panel $(b)$ is model (4) with $\sigma_{\mathrm{M}}=0.20, F=0.75$, panel (c) is model (5) with $\sigma_{\mathrm{M}}=0.75, F=0.75$, panel $(d)$ is model (6) with $\sigma_{\mathrm{M}}=1.45, F=0.75$, panel (e) is model (7) with $\sigma_{\mathrm{M}}=1.45, F=0.85$ and panel $(f)$ is model (8) with $\sigma_{\mathrm{M}}=0.05, F=0.75$.

\section{COMPARISON WITH OBSERVATIONS}

The goal of the parameter search presented here has been primarily to populate an initial portion of the parameter space where solutions that are good candidates for the application to real sources reside. As shown in Fig. 11, we retrieve solutions that have bulk Lorentz factors at the MFP ranging from 1-11 and MFP's height that spans 4 orders of magnitude $\left(\sim 10^{3}-10^{7} r_{\mathrm{g}}\right)$. Moreover, we find jets with different initial conditions exhibiting a large range of magnetic-tothermal energy ratios and different degrees of winding at the base. The set of initial conditions, the positions of the singular points, velocity, density and magnetic field profiles 

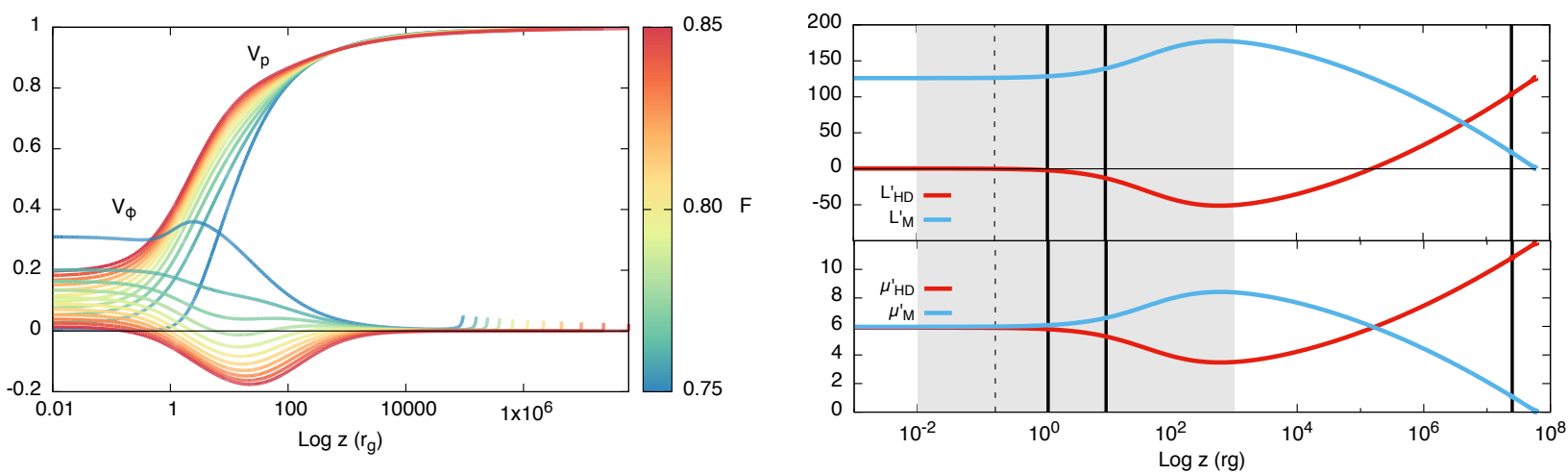

Figure 9. Left panel: Toroidal and poloidal velocities in units of $c$ for a series of solutions obtained for increasing $F$. The solution of this series with higher $F$ is model (7) in Tab. 2. Right panel:. Angular momentum and energy components defined in equations 8 and 9 for model (7). The vertical solid lines are the MSP, AP and MFP of this solution, while the dashed line marks the height of the jet where $L_{\mathrm{HD}}^{\prime}$ becomes negative. The shaded grey areas are shown as a comparison with the range considered in figure 2 of Cayatte et al. (2014).
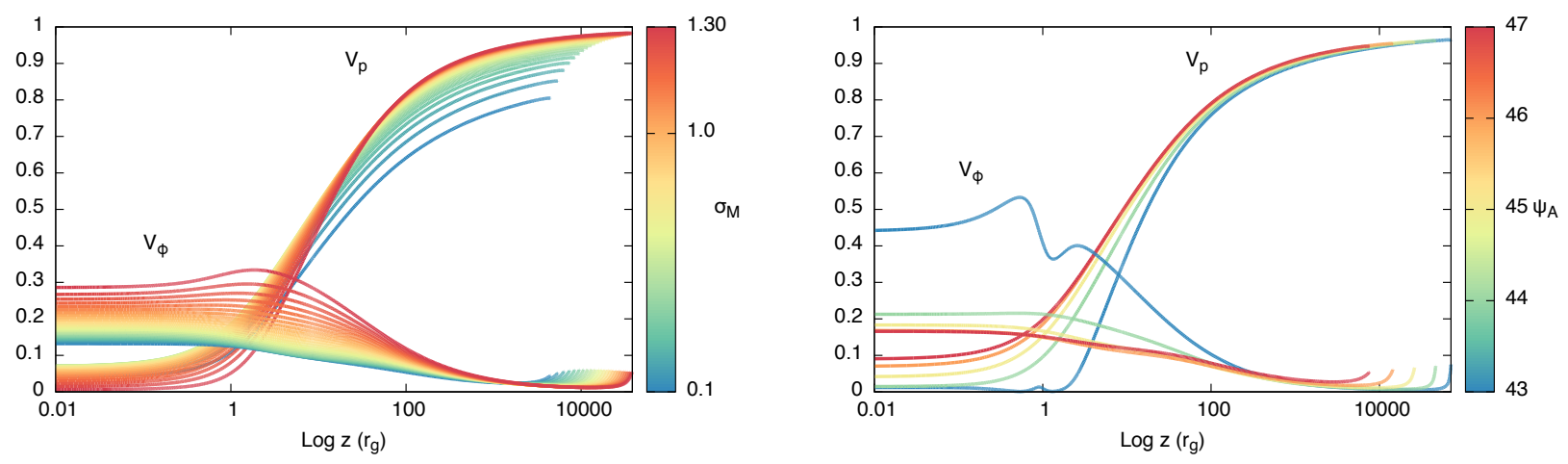

Figure 10. Left panel: Toroidal and poloidal velocities in units of $c$ for a series of solutions obtained increasing $\sigma_{\mathrm{M}}$ at fixed $\psi_{\mathrm{A}}=46^{\circ}$. Right panel: Same figure as the left panel, but for increasing $\psi_{\mathrm{A}}$ at fixed $\sigma_{\mathrm{M}}=0.55$. The other fixed parameters for both plots are $\theta_{\mathrm{A}}=60^{\circ}, F=0.75, \Gamma=4 / 3$ and $\varpi_{\mathrm{A}}=15$.

can be either directly compared to observations or can be used to provide constraints for the calculation of the emission and the polarisation degree expected from a particular jet configuration.

For instance, the MFP is the location where the jet streamlines start to recollimate and the flow downstream of this region loses causal contact with the upstream flow. These are favourable conditions for the onset of a shock, while maintaining the structure upstream intact (Polko et al. 2010). If, furthermore, we assume that there is a correspondence between the MFP and the frequency of the jet break, the self-absorption turnover in the synchrotron spectrum from the region where particle acceleration initiates in the jet (Markoff et al. 2001, 2005; Markoff 2010), we can use observational constraints on the position of the jet break to determine the best jet solution within the set that we have.

In AGN, the equivalent jet break can be at large offsets from the $\mathrm{BH}\left(>10^{4} r_{\mathrm{g}}\right)$ and there could be substantial contamination in the determination of the jet break by the interaction of the jet with the environment (Russell et al. 2015) or the scenario could be further complicated by a multiple-flow dynamics (see Meier 2003 for a theoretical motivation of structured jets and e.g. Giroletti et al. 2004; Harris \& Krawczynski 2006; Meyer et al. 2013 for observa- tional evidence on the radial structure of the jets of M87). High-resolution observations can now resolve the details of the jet structure in AGN down to a few gravitational radii. Radio interferometry and high-resolution optical and X-ray observations unveiled bright subfeatures, usually referred to as knots, which are identified with shocks and particle acceleration. Knots can occur everywhere along the jet. Some of them are attributed to internal shocks caused for example by the interactions of the jet with a dense external cloud (Mendoza \& Longair 2001), variations in the injected mass and velocity (Rees \& Meszaros 1994; Malzac 2014) or magnetic recollimation (Markoff 2010). However, knots that occur at larger distances from the black holes can also be produced by self-collimation of the streamlines (Polko et al. 2014) or a change in the ISM pressure profile (Nakamura \& Asada 2013).

The jet of M87, for example, is one of the main targets for this type of study. It exhibits a complex pattern of knots with different values and orientations of the proper motion. In particular, the HST-1 knot has received significant interest over time. HST-1 is located approximately at $2-5 \times 10^{5} r_{\mathrm{g}}$, which also corresponds to the region where the Bondi radius resides $\left(r_{\mathrm{B}} \sim 3.5 \times 10^{5} r_{\mathrm{g}}\right.$ for $M_{\mathrm{BH}}=$ $\left.6 \times 10^{9} M_{\odot}\right)$. The Bondi radius marks the volume where 

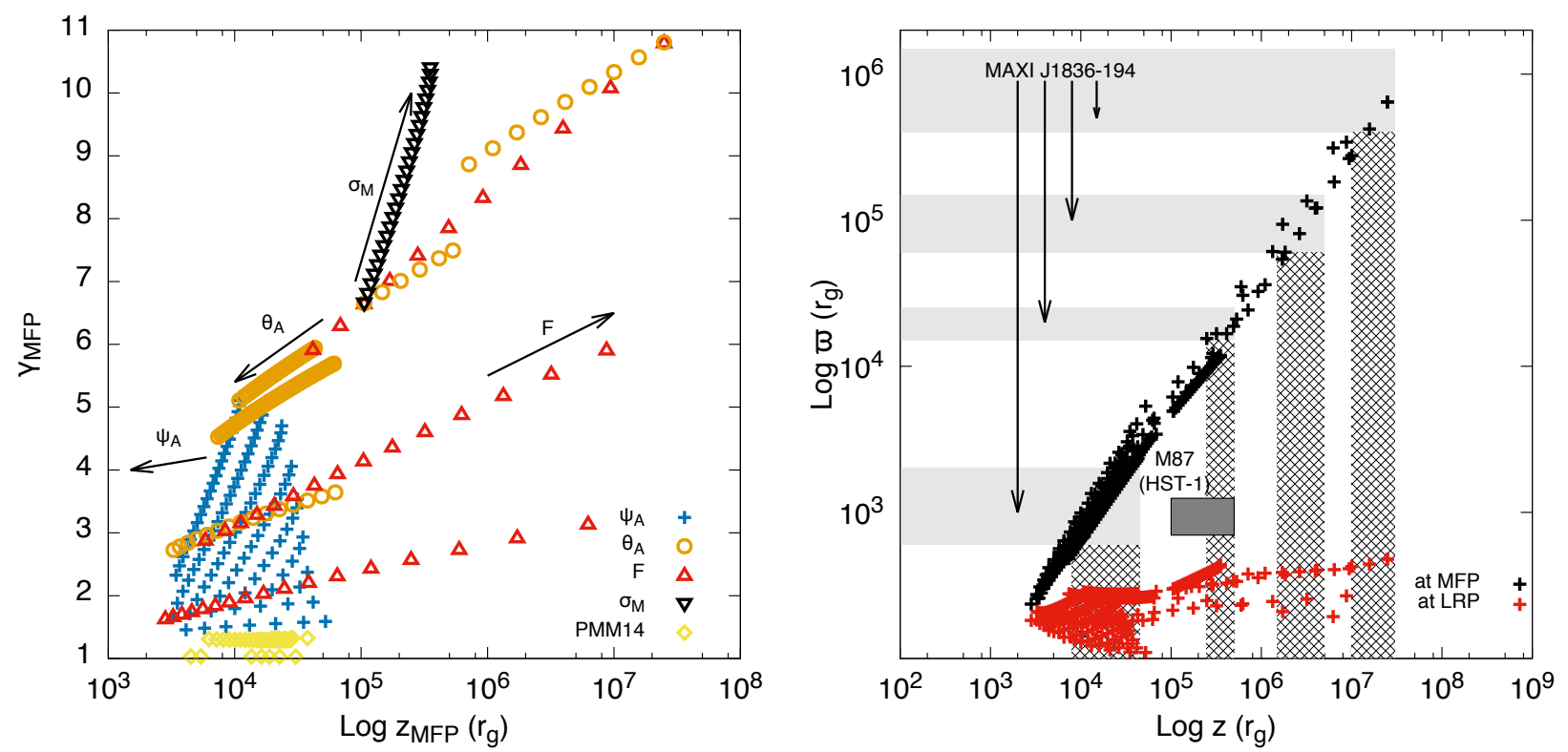

Figure 11. Left panel: Distribution of the Lorentz factors at MFP of all solutions, as a function of the height of the MFP. The blue crosses are solutions belonging to the 2-dimensional search varying $\psi_{\mathrm{A}}$ for given values of $\sigma_{\mathrm{M}}$. The other symbols are for solutions found by varying $\theta_{\mathrm{A}}$ (orange hollow circles), $\sigma_{\mathrm{M}}$ (black hollow downward triangles), $F$ (red hollow triangles) and PMM14 with correct gravity terms (yellow hollow diamonds). The arrows mark the direction of increasing values of the given parameter. Right panel: Distribution of the same collection of solutions shown in the the left panel with respect to cylindrical radius versus height of the MFP (black crosses) and of the last recollimation point (LRP, red crosses). The shaded light grey areas corresponds to the radial coordinates of the jet at the jet break with error bars reported by Russell et al. (2014) for the XRB MAXI J1836-194. Solutions are present within each region where the jet break has been seen (grey areas). The areas with the oblique-line pattern denotes the predicted heights for the jet break. The dark grey rectangle is the area where the knot HST-1 in the jet of M87 is estimated to be by Asada \& Nakamura (2012). The authors pointed out that the jet cross section at HST-1 is smaller than the one predicted by conical and parabolic jet models. We note that HST-1 could be tracing an intermediate location between the MFP and LRP, where the jet is rapidly collimating towards the axis.

the gravitational potential of the black hole dominates over the thermal energy of the gas contained within this volume. The density profile within and outside the Bondi radius can be substantially different, hence the jet could experience a steep gradient in the external pressure which could induce a recollimation shock (for observational evidence see Russell et al. 2015 and for recent simulations see Barniol Duran et al. 2016).

Asada \& Nakamura (2012) and Asada et al. (2014) show that the M87 jet maintains a parabolic profile up to HST-1. The authors show that this is evidence of the extension of the acceleration and collimation region of the jet of M87 up to the end of the sphere of influence of the black hole.

If we assume then that the HST-1 knot in the jet of M87 coincides with a recollimation shock and acceleration region in the jet produced by self-collimation, we can identify it with the MFP or LRP. In all the solutions presented in this paper we see that the jet continues recollimating downstream of the MFP and we possibly see conditions that could lead to a shock due to the compression of the gas in correspondence of the last recollimation point. The flow could become causally disconnected but still remain smooth through the MFP, then undergoing a shock only when it is over-compressed. The distance between the MFP and LRP is relatively large in AGN so that it can be resolved by current high-resolution observations.

Using the interval given by Asada \& Nakamura (2012) for the width of the jet at HST-1 (dark grey area in Fig. 11, right panel), we see that the estimated position of HST-1 falls in between the MFP and the last recollimation point of the same jet solutions. HST-1 has a smaller cross-section with respect to a canonical paraboloidal jet, probably indicating that the streamlines are converging towards the jet axis and a shock could take place soon after the MFP.

Although the identification of HST-1 with a self-collimation shock is tempting, also the change in the external pressure could influence the collimation of the jet and the shock formation due to the proximity of the Bondi radius. There is also increasing evidence that a first recollimation occurs within the first few tens of $r_{\mathrm{g}}$ (Prieto et al. 2016; Hada et al. 2016), suggesting a more complex scenario.

In XRBs, the position of the jet break has been identified in a few cases in the range of of $10-1000 r_{\mathrm{g}}$. The turnover has been directly observed only in three cases (GX339-4 by Corbel \& Fender 2002, MAXI J1836-194 by Russell et al. 2014 and 4U 0614+091 by Migliari et al. 2006). Russell et al. (2014) show that the jet break shifts by $\sim 3$ orders of magnitude in frequency during state transition in the source MAXI J1836-194. The estimated width of the acceleration region also presents large variations during the transition of the source, decreasing as the jet break moves closer to the BH. With the solutions that we have collected so far, we cover the full range of the jet widths seen in MAXI J1836-194 (see right panel of Fig. 11). Solutions found within the grey areas in Fig. 11 not only can reproduce the inferred widths of the jet at the location of the shock, but they give 
a full set of predicted physical properties of the corresponding jet, such as the height of the shock (identified with the MFP), the bulk velocity of the flow and the magnetic field morphology at any point of the jet.

Empirically constraining some of the jet properties, such as the Lorentz factor of the flow, indirectly from observations is very difficult. For instance, XRBs are thought to have mildly relativistic jets with $2<\gamma_{\mathrm{j}}<5$, while the Lorentz factor of AGN could span a larger range. However, recent works have shown that the range of indirectly inferred Lorentz factors for XRBs could be biased towards the lower end (Fender 2003; Miller-Jones et al. 2006) and in fact be the same of $\operatorname{AGN}\left(\gamma_{\mathrm{j}} \sim 1-50\right)$. With the solutions that we present here, the velocity profile is determined as part of a jet solution, and can be constrained together with other observables.

With the increasing attention on polarisation studies and the growing number of current and upcoming devoted facilities, particularly the first X-ray polarimeter IXPE (Imaging X-ray Polarimetry Explorer, Weisskopf et al. 2016), it is crucial to properly model the jet magnetic field and the emission produced by such configuration of fields and particles. Indeed, as shown by various works, e.g. Perlman et al. (1999); Avachat et al. (2016); Russell \& Shahbaz (2014), multiwavelength polarisation studies can help constrain the magnetic field morphology over large scales. The parameter degeneracy in the evaluation of the spectral energy distribution of the given source is also greatly reduced by polarisation measurements. The need of a more detailed MHD treatment of the modelling of jets, which could be used to infer their emission is therefore compelling.

\section{SUMMARY AND CONCLUSION}

We presented here a new numerical scheme to solve the equations for stationary, axisymmetric, radially selfsimilar, relativistic MHD jets. Such class of jet models have been studied extensively over the last three decades and adopted in numerical studies and simulations of the acceleration/collimation region of jets with various applications, from gamma-ray bursts to young stellar objects. In the case of semi-analytical models of PMM10,PMM13,PMM14, the numerical approach adopted for the integration of the equations describing the outflow was heavily affecting the efficiency of the parameter space search. Moreover, in the most recent development, the gravity terms in PMM14 were calculated with excessive approximations, which led to extra inaccuracies in the results. We avoid inconsistent approximations and also use the correct derivatives of the gravitational potential with respect to $\theta$ : in this way we use fully self-consistent gravity terms. With this new setup, we are able to recover and compare the solutions found in PMM14 as a test for our algorithm. The consistency between the equations affects the parameters of the solutions and, consequently, the observables that we derive from them. Once the extra approximations are removed, the contribution of gravity is stronger and this leads to a more restricted range of radii within which self-similarity holds for a given set of outflow parameters.

We were able to find solutions corresponding to single streamlines in large parts of the parameter space. The multiple jet configurations retrieved from the initial search presented in this paper are shown to be diverse in geometrical and dynamical properties, such as magnetic-to-thermal energy ratio at the jet foot point, bulk velocity, morphology of the streamlines and the position of the MFP. However, the parameter space is not continuous and the exploration along a specific direction, i.e. varying the value of a given parameter, can be interrupted due to the approach of physical limits or the break-down of our assumptions. Solutions can cease to exist because of the jet velocity approaching the speed of light around the MFP or due to the impossibility of launching the jet because of a too small energy reservoir at the base. In the latter case, the flow keeps circulating around the jet axis and never gains enough poloidal velocity to be slung out. Alternatively, solutions cannot be found when the LRP takes place before the MFP, while our method is constructed so to cross all three singular points.

By studying the evolution of the energy and the velocity components for all solutions, we encounter both cold and hot relativistic jets, the latter configurations exhibiting counterrotation at distances $\sim 0.1-10^{5} \mathrm{r}_{\mathrm{g}}$. With the exploration of the parameter space that we have conducted, we are able to cover a large area of the parameter space and to move towards a desired direction, e.g. higher bulk Lorentz factors, by making use of the trends that we observed so far.

Taking into account the large variety of jet configurations and dynamics that we can obtain with our numerical scheme, the extension to other accreting objects, e.g. young stellar objects, is within the capabilities of this model. Future steps will be taken to increase the density of the solution grid and cover larger volumes of the parameter space. This mapping of the parameter space is ultimately aimed at providing a dynamic and flexible base for future coupling with a radiative code. That, in turn, will eventually allow data-fitting of both XRBs and AGN, which will be the subject of forthcoming papers. We are also aware of the need to benchmark the results of our method to fully numerical simulations to properly quantify the limits of our approach.

\section{ACKNOWLEDGEMENT}

CC is supported by the Netherlands Organisation for Scientific Research (NWO), grant Nr. 614.001.209. SM acknowledges support from NWO VICI grant Nr. 639.043.513. YC is supported by the European Union's Horizon 2020 research and innovation programme under the Marie SklodowskaCurie Global Fellowship grant agreement No 703916. 


\section{APPENDIX A: DEFINITIONS OF THE EQUATIONS}

We present here the system of equations (1)-(4) in its full form as given in PMM14 and we report the changes in the function of the gravitational pseudo-potential.

The Bernoulli equation and the transfield equation assume the same form when we write them down in terms of the functions $A_{i}, B_{i}, C_{i}$ with $i=1,2$

$$
\begin{aligned}
& A_{1} \frac{d M^{2}}{d \theta}+B_{1} \frac{d \psi}{d \theta}=C_{1} \\
& A_{2} \frac{d M^{2}}{d \theta}+B_{2} \frac{d \psi}{d \theta}=C_{2}
\end{aligned}
$$

where

$$
\begin{aligned}
& A_{1}=\frac{\cos ^{3}(\theta+\psi)}{\sin ^{2} \theta \sin (\theta+\psi)}\left[\left(\frac{\mu x_{\mathrm{A}}^{2}}{F \sigma_{\mathrm{M}}}\right)^{2} \frac{x_{\mathrm{A}}^{2} M^{2}}{G^{2}} \frac{\left(1-G^{2}\right)^{2}}{\left(1-M^{2}-x^{2}\right)^{3}}-\left(\frac{\xi x_{\mathrm{A}}^{2}}{F \sigma_{\mathrm{M}}}\right)^{2} \frac{(\Gamma-1)(\xi-1)}{(2-\Gamma) \xi+\Gamma-1} \frac{1}{M^{2}}\right]+\frac{M^{2}}{G^{4}} \frac{\cos (\theta+\psi)}{\sin (\theta+\psi)} \\
& B_{1}=\frac{M^{4}}{G^{4}} \\
& C_{1}=\frac{\cos (\psi) \cos ^{2}(\theta+\psi)}{\sin ^{3} \theta \sin (\theta+\psi)}\left\{\left(\frac{\mu x_{\mathrm{A}}^{2}}{F \sigma_{\mathrm{M}}}\right)^{2} \frac{\left(1-M^{2}-x_{\mathrm{A}}^{2}\right)^{2}}{\left(1-M^{2}-x^{2}\right)^{2}}-\left(\frac{\xi x_{\mathrm{A}}^{2}}{F \sigma_{\mathrm{M}}}\right)^{2}+\left(\frac{\mu x_{\mathrm{A}}^{2}}{F \sigma_{\mathrm{M}}}\right)^{2} \frac{2 x^{2}}{G^{4}\left(1-M^{2}-x^{2}\right)^{3}}\left[G^{4}\left(1-M^{2}-x_{\mathrm{A}}^{2}\right)^{2}+C_{1}^{+}\right.\right. \\
& \left.\left.-x^{2}\left(G^{2}-M^{2}-x^{2}\right)^{2}-\left(1-M^{2}-x^{2}\right) G^{2}\left(1-x_{\mathrm{A}}^{2}\right)\left(G^{2}-M^{2}-x^{2}\right)\right]\right\} \\
& A_{2}=\sin (\theta+\psi) \cos (\theta+\psi)\left[\left(\frac{\mu x_{\mathrm{A}}^{2}}{F \sigma_{\mathrm{M}}}\right)^{2} \frac{x^{2}\left(1-G^{2}\right)^{2}}{\left(1-M^{2}-x^{2}\right)^{3}}-\left(\frac{\xi x_{\mathrm{A}}^{2}}{F \sigma_{\mathrm{M}}}\right)^{2} \frac{(\Gamma-1)(\xi-1)}{(2-\Gamma) \xi+\Gamma-1} \frac{G^{4}}{M^{4}}\right] \\
& B_{2}=\sin ^{2} \theta\left[\frac{\left(1-x^{2}\right)}{\cos ^{2}(\theta+\psi)}-M^{2}\right] \\
& C_{2}=\frac{\cos (\psi) \sin (\theta+\psi)}{\sin \theta} \frac{G^{4}}{M^{2}}\left[\left(\frac{\mu x_{\mathrm{A}}^{2}}{F \sigma_{\mathrm{M}}}\right)^{2} \frac{\left(1-M^{2}-x_{\mathrm{A}}^{2}\right)^{2}}{\left(1-M^{2}-x^{2}\right)^{2}}-\left(\frac{\xi x_{\mathrm{A}}^{2}}{F \sigma_{\mathrm{M}}}\right)^{2}+\left(\frac{\mu x_{\mathrm{A}}^{2}}{F \sigma_{\mathrm{M}}}\right)^{2} \frac{2 x^{2}}{G^{2}\left(1-M^{2}-x^{2}\right)^{3}} M^{2}\left(1-G^{2}\right)\left(1-M^{2}-x_{\mathrm{A}}^{2}\right)\right] \\
& +\frac{2}{M^{2}}\left(\frac{x^{2}}{F \sigma_{\mathrm{M}}}\right)^{2} \frac{(\Gamma-1)(F-2) \xi(\xi-1)}{\Gamma}+\left[2 x^{2}+\left(1-M^{2}-x^{2}\right)\right] \frac{\cos (\psi) \sin \theta \sin (\theta+\psi)}{\cos ^{2}(\theta+\psi)} \\
& +\frac{\sin ^{2} \theta}{\cos ^{2}(\theta+\psi)}\left(F-2-F x^{2}+x^{2}\right)+\left(\frac{\mu x_{\mathrm{A}}^{2}}{F \sigma_{\mathrm{M}}}\right)^{2} \frac{x^{2}\left[M^{2}\left(1-G^{2}\right)^{2}(F-1)-\left(G^{2}-M^{2}-x^{2}\right)^{2}\right]}{M^{2}\left(1-M^{2}-x^{2}\right)^{2}}+C_{2}^{+} \text {. }
\end{aligned}
$$

where $\mu=\mu^{\prime} /\left(1-P_{\mathrm{g}}\right)$. The last terms in the $C_{i}$ are the gravity terms and they can be written schematically as

$$
C_{1}^{+}=f_{1}\left(P_{\mathrm{g}}\right) C_{1}^{+, \text {noP }}, \quad C_{2}^{+}=f_{2}\left(P_{\mathrm{g}}\right) C_{2}^{+, n o P}
$$

where

$C_{1}^{+, n o P}=\frac{\mu^{2} x_{\mathrm{A}}^{4}}{F^{2} \sigma_{\mathrm{M}}^{2}} \frac{\cos ^{2}(\theta+\psi)}{\sin ^{2} \theta}\left[\frac{G^{2}\left(1-M^{2}-x_{\mathrm{A}}^{2}\right)^{2}-x_{\mathrm{A}}^{2}\left(G^{2}-M^{2}-x^{2}\right)^{2}}{G^{2}\left(1-M^{2}-x^{2}\right)^{2}}\right]$

$C_{2}^{+, n o P}=\left\{\left(\frac{x^{4}}{F^{2} \sigma_{\mathrm{M}}^{2}}\right)\left[\frac{\mu^{2}}{M^{2}} \frac{\left(1-M^{2}-x_{\mathrm{A}}^{2}\right)^{2}}{\left(1-M^{2}-x^{2}\right)^{2}}+\frac{\mu^{2} x^{2}}{2 G^{4}} \frac{\left(1-G^{2}\right)^{2}}{\left(1-M^{2}-x^{2}\right)^{2}}-\frac{\Gamma-1}{\Gamma} \frac{\xi(\xi-1)}{M^{2}}\right]+\frac{1}{2}\left(1+x^{2}\right) \frac{\sin ^{2} \theta}{\cos ^{2}(\theta+\psi)}\right\} \cos ^{2}(\theta+\psi)$

The functions $f_{i}\left(P_{\mathrm{g}}\right)(i=1,2)$ of the potential can be evaluated in a Newtonian or a Paczyńsky-Wiita scenario. The choice of using the Paczyńsky-Wiita pseudo-potential is easily motivated. The Paczyńsky-Wiita potential diverges at the Schwarzschild radius, $r_{\mathrm{s}}=2 r_{\mathrm{g}}$, so it mimics general relativity with the advantage of maintaining a newtonian formalism. If we define the potential as

$$
P_{\mathrm{g}}=\left\{\begin{array}{cll}
\Phi, & \text { if } & \text { Newton } \\
\frac{\Phi}{(1-2 \Phi)}, & \text { if } & \text { Paczyńsky-Wiita }
\end{array}\right.
$$

where $\Phi=\sin (\theta) /\left(\varpi_{A} G\right)=r_{\mathrm{g}} / r, \varpi_{\mathrm{A}}$ is in units of $r_{\mathrm{g}}=\mathcal{G M} / c^{2}$. Defining the following function

$$
\mathcal{F}\left(P_{\mathrm{g}}\right)=\left\{\begin{array}{lll}
1, & \text { if } & \text { Newton } \\
1+2 P_{\mathrm{g}}, & \text { if } & \text { Paczyńsky-Wiita, }
\end{array}\right.
$$

the gravity terms appear in the convenient form (A8) with

$$
f_{1}\left(P_{\mathrm{g}}\right)=-P_{\mathrm{g}} \frac{\mathcal{F}\left(P_{\mathrm{g}}\right)}{\left(1-P_{\mathrm{g}}\right)}, \quad f_{2}\left(P_{\mathrm{g}}\right)=P_{\mathrm{g}} \mathcal{F}\left(P_{\mathrm{g}}\right) .
$$

MNRAS 000, 000-000 (0000) 
It is worth noting that the equations (A13) differ from PMM14 which in our notation are

$$
f_{1}\left(P_{\mathrm{g}}\right)=-P_{\mathrm{g}}, \quad f_{2}\left(P_{\mathrm{g}}\right)=P_{\mathrm{g}} .
$$

Rearranging the terms in Eq. (A1)-(A2), we obtain two differential equations, (1) and (5), for $M^{2}$ and $\psi$.

At the Alfvén point, all the equations in the system of equations (1)-(4) can be regularized with De L'Hôpital rule, starting from the quantities $\theta=\theta_{\mathrm{A}}, \psi=\psi_{\mathrm{A}}, x=x_{\mathrm{A}}$ and $\xi=\xi_{A}$, as follows:

$$
\begin{aligned}
& G_{\mathrm{A}}=1, \quad M_{\mathrm{A}}^{2}=1-x_{\mathrm{A}}^{2} \\
& \sigma=\frac{x_{\mathrm{A}}^{2}-x^{2}}{1-M^{2}-x_{\mathrm{A}}^{2}} \longrightarrow \sigma_{\mathrm{A}}=\frac{2 x_{\mathrm{A}}^{2} \cos \psi_{\mathrm{A}}}{p_{\mathrm{A}} \sin \theta_{\mathrm{A}} \cos \left(\theta_{\mathrm{A}}+\psi_{\mathrm{A}}\right)}, \\
& \left(\frac{1-M^{2}-x_{\mathrm{A}}^{2}}{1-M^{2}-x}\right)_{\mathrm{A}}=\frac{1}{\sigma_{\mathrm{A}}+1}, \quad\left(\frac{1-G^{2}}{1-M^{2}-x}\right)_{\mathrm{A}}=\frac{\sigma_{\mathrm{A}} / x_{\mathrm{A}}^{2}}{\sigma_{\mathrm{A}}+1}, \quad\left(\frac{G^{2}-M^{2}-x^{2}}{1-M^{2}-x}\right)_{\mathrm{A}}=\frac{x_{\mathrm{A}}^{2}-\left(1-x_{\mathrm{A}}^{2}\right) \sigma_{\mathrm{A}}}{x_{\mathrm{A}}^{2}\left(\sigma_{\mathrm{A}}+1\right)}, \\
& \left.\frac{d M^{2}}{d \theta}\right|_{\mathrm{A}}=p_{\mathrm{A}},\left.\quad \frac{d G^{2}}{d \theta}\right|_{\mathrm{A}}=\frac{2 \cos \psi_{\mathrm{A}}}{\sin \theta_{\mathrm{A}} \cos \left(\theta_{\mathrm{A}}+\psi_{\mathrm{A}}\right)}
\end{aligned}
$$

where $p_{\mathrm{A}}$ is given by the Alfvén regularity condition (ARC) as described in Polko et al. (2010) and Polko et al. (2014). The $\mathrm{ARC}$ is obtained by calculating the wind equation at the Alfvén point using the De L'Hôpital rule (Eq. A15-A18). Therefore we have

$$
\left.\frac{d M^{2}}{d \theta}\right|_{\mathrm{A}}=\frac{B_{2, \mathrm{~A}} C_{1, \mathrm{~A}}-B_{1, \mathrm{~A}} C_{2, \mathrm{~A}}}{A_{1, \mathrm{~A}} B_{2, \mathrm{~A}}-A_{2, \mathrm{~A}} B_{1, \mathrm{~A}}},
$$

that, after recasting terms, becomes

$$
\begin{aligned}
0=- & \mathscr{G}_{\mathrm{A}}+2 \frac{\Gamma-1}{\Gamma} \frac{F-2}{F^{2} \sigma_{\mathrm{M}}^{2}} \xi_{\mathrm{A}}\left(\xi_{\mathrm{A}}-1\right)\left(1-x_{\mathrm{A}}^{2}\right) x_{\mathrm{A}}^{4}+\frac{\sin ^{2}\left(\theta_{\mathrm{A}}\right)\left(1-x_{\mathrm{A}}^{2}\right)^{2}}{\cos ^{2}\left(\psi_{\mathrm{A}}+\theta_{\mathrm{A}}\right)}\left[\left(1-x_{\mathrm{A}}^{2}\right)(F-1)-1\right] \\
& +\frac{\mu^{2} x_{\mathrm{A}}^{2}}{F^{2} \sigma_{\mathrm{M}}^{2}}(F-1) \sigma_{\mathrm{A}}^{2} \frac{\left(1-x_{\mathrm{A}}^{2}\right)^{2}}{\left(\sigma_{\mathrm{A}}+1\right)^{2}}-\frac{\mu^{2} x_{\mathrm{A}}^{2}}{F^{2} \sigma_{\mathrm{M}}^{2}} \frac{1-x_{\mathrm{A}}^{2}}{\left(\sigma_{\mathrm{A}}+1\right)^{2}}\left[x_{\mathrm{A}}^{2}-\sigma_{\mathrm{A}}\left(1-x_{\mathrm{A}}^{2}\right)\right]^{2} \\
& +2 \frac{\cos \left(\psi_{\mathrm{A}}\right) \sin \left(\theta_{\mathrm{A}}\right) \sin \left(\psi_{\mathrm{A}}+\theta_{\mathrm{A}}\right)}{\cos ^{2}\left(\psi_{\mathrm{A}}+\theta_{\mathrm{A}}\right)} x_{\mathrm{A}}^{2}\left(1-x_{\mathrm{A}}^{2}\right)^{2} \frac{\sigma_{\mathrm{A}}+1}{\sigma_{\mathrm{A}}}
\end{aligned}
$$

where the gravity term is given by

$$
\begin{gathered}
\mathscr{G}_{\mathrm{A}}=C_{1, \mathrm{~A}}^{+} B_{2, \mathrm{~A}}-C_{2, \mathrm{~A}}^{+} B_{1, \mathrm{~A}}=f_{1, \mathrm{~A}}\left(P_{\mathrm{g}}\right)\left(1-x_{\mathrm{A}}^{2}\right) \sin \left(\theta_{\mathrm{A}}\right)^{2} \tan \left(\psi_{\mathrm{A}}+\theta_{\mathrm{A}}\right)^{2}\left\{\left(\frac{\xi_{\mathrm{A}} x_{\mathrm{A}}^{2}}{F \sigma_{\mathrm{M}}}\right)^{2} \frac{\cos ^{2}\left(\psi_{\mathrm{A}}+\theta_{\mathrm{A}}\right)}{\sin ^{2}\left(\theta_{\mathrm{A}}\right)}+\left(1-x_{\mathrm{A}}^{2}\right)^{2}\right\} \\
-f_{2, \mathrm{~A}}\left(P_{\mathrm{g}}\right)\left(1-x_{\mathrm{A}}^{2}\right)^{2} \cos ^{2}\left(\theta_{\mathrm{A}}+\psi_{\mathrm{A}}\right)\left\{\left(\frac{\mu_{\mathrm{A}} x_{\mathrm{A}}^{2}}{F \sigma_{\mathrm{M}}}\right)^{2} \frac{2 x_{\mathrm{A}}^{2}+\left(1-x_{\mathrm{A}}^{2}\right) \sigma_{\mathrm{A}}^{2}}{2 x_{\mathrm{A}}^{2}\left(1-x_{\mathrm{A}}^{2}\right)\left(\sigma_{\mathrm{A}}+1\right)^{2}}\right. \\
\left.-\frac{x_{\mathrm{A}}^{4}}{\left(F \sigma_{\mathrm{M}}\right)^{2}} \frac{\Gamma-1}{\Gamma} \frac{\xi_{\mathrm{A}}\left(\xi_{\mathrm{A}}-1\right)}{1-x_{\mathrm{A}}^{2}}+\frac{1+x_{\mathrm{A}}^{2}}{2} \frac{\sin ^{2}\left(\theta_{\mathrm{A}}\right)}{\cos ^{2}\left(\psi\left(\theta_{\mathrm{A}}+\psi_{\mathrm{A}}\right)\right)}\right\}
\end{gathered}
$$

with $f_{1, \mathrm{~A}}\left(P_{\mathrm{g}}\right)$ and $f_{2, \mathrm{~A}}\left(P_{\mathrm{g}}\right)$ are calculated with $\Phi_{\mathrm{A}}=\sin \left(\theta_{\mathrm{A}}\right) / \varpi_{\mathrm{A}}$.

\section{APPENDIX B: DERIVATION OF THE PSEUDO-POTENTIAL FUNCTIONS IN THE GRAVITY TERMS}

Here we give the details of the derivation of the functions A13. First and foremost, we need to carry out the derivative of the gravitational potential to include them into the system (A1)-(A2). We take the derivative with respect to $r$ (since the gravitational potential depends solely on $r$ ), and then, applying the assumptions of axisymmetry and self-similarity, we obtain the corresponding forms in $\theta$. Since we want to keep the flexibility of changing between the Newtonian potential and the Paczyńsky-Wiita pseudo-potential, we differentiate both $P_{\mathrm{gs}}$ as follows

$$
\frac{\partial P_{\mathrm{g}}}{\partial r}=\left\{\begin{array}{lll}
-\frac{P_{\mathrm{g}}}{r}, & \text { if } & \text { Newton } \\
-\frac{P_{\mathrm{g}}}{r}\left(1+2 P_{\mathrm{g}}\right), & \text { if } & \text { Paczyńsky-Wiita }
\end{array}\right.
$$

or, in a more compact way

$$
\frac{\partial P_{\mathrm{g}}}{\partial r}=-\frac{P_{\mathrm{g}}}{r} \mathcal{F}\left(P_{\mathrm{g}}\right)
$$

where $\mathcal{F}\left(P_{\mathrm{g}}\right)$ is defined as in A12. Now, we need to calculate again the gravity terms in both (A1)-(A2). Let's start from the gravity term in the transfield equation in the general form equivalent to Eq. 8 of PMM14

$$
-\left(\gamma \rho_{0}+\mathcal{E} / c^{2}\right) c^{2} \nabla P_{\mathrm{g}} \cdot \hat{n}
$$


where $\mathcal{E}=\gamma(\gamma-1) \rho_{0} c^{2}+P\left(\gamma^{2} \Gamma /(\Gamma-1)-1\right)+\left(B^{2}+E^{2}\right) /(8 \pi)$ is the energy density. Since we are calculating the derivative with respect to $r$ and $\hat{n}=\cos (\theta+\psi) \hat{r}-\sin (\theta+\psi) \hat{\theta}$ ( $\hat{n}$ is the unit vector perpendicular to the field line, towards the jet axis, as defined in Section 2.1 and 3.1 of VK03), then $\nabla P_{\mathrm{g}} \cdot \hat{n}=\cos (\theta+\psi) \partial_{r} P_{\mathrm{g}}$. The derivative of $P_{\mathrm{g}}$ with respect to $r$ is given by Eq. (B1).

Recasting the term in parenthesis in Eq. (B3) in dimensionless units (see Appendix D and VK03), applying self-similarity $(\partial / \partial \phi=0)$ and using $\varpi=r \sin \theta$ and $G \equiv \varpi / \varpi_{\mathrm{A}}$, we have

$$
\nabla P_{\mathrm{g}} \cdot \hat{n}=\cos (\theta+\psi) \frac{\sin \theta}{\varpi} P_{\mathrm{g}} \mathcal{F}\left(P_{\mathrm{g}}\right)
$$

therefore the gravity term in the transfield equation is

$$
-\left\{\frac{B_{0}^{2} \alpha^{F-2}}{4 \pi \varpi G^{4}} \frac{\sin \theta}{\cos (\theta+\psi)}\right\}\left[\cos ^{2}(\theta+\psi) P_{\mathrm{g}} \mathcal{F}\left(P_{\mathrm{g}}\right)\right] \frac{C_{2}^{+, n o P}}{\cos ^{2}(\theta+\psi)} .
$$

Similarly, we take the derivative of the Bernoulli equation (Eq. 4 in PMM14) with respect to $r$ and, using Eq. 6, we find

$$
\frac{d \mu}{d r} \equiv \mu^{\prime 2} \frac{d}{d r}\left[\frac{1}{\left(1-P_{\mathrm{g}}\right)^{2}}\right]=-2 \mu^{2} \frac{P_{\mathrm{g}}}{\left(1-P_{\mathrm{g}}\right)} \mathcal{F}\left(P_{\mathrm{g}}\right) \frac{1}{r}
$$

which once being included in the derivative of the Bernoulli equation under the self-similar assumption becomes

$$
\left\{-2 \tan (\theta+\psi) G^{6} F^{2} \sigma_{\mathrm{M}}^{2}\left(1-M^{2}-x^{2}\right)^{2} \sin ^{2} \theta\right\}\left[-2 \mu^{2} \frac{P_{\mathrm{g}}}{\left(1-P_{\mathrm{g}}\right)} \mathcal{F}\left(P_{\mathrm{g}}\right)\right] C_{1}^{+, n o P} .
$$

It is worth noting that the scaling of both equations B5 and B7 (terms in curly brackets) can be simplified from all functions $A, B, C$, as done by PMM14.

\section{APPENDIX C: INITIAL CONDITIONS}

Once we find the initial conditions for $M^{2}$ and $G^{2}$ and their derivatives at the three critical points, we perform the integration from each point with an adaptive stepsize Runge-Kutta scheme as described in Press et al. (1993, hereafter NR93), until we

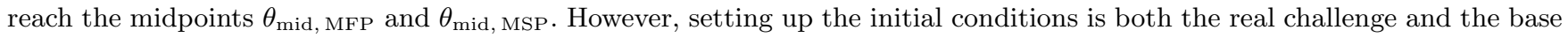
for the robustness of our scheme.

As we mentioned in the Sect.3, given the full set of parameters, the initial conditions at the AP are readily calculated. The only numerical step is finding $d M^{2} / d \theta$. The derivative of $M^{2}$ at Alfvén is commonly referred to as $p_{\mathrm{A}}$ and can be found from the Alfvén regularity condition as described in Polko et al. $(2010,2014)$ and in eq. A20. Now the integral of motion $\mu^{\prime}$ can also be calculated (see Eq. 6) and all the other quantities can be analytically calculated at the Alfvén point.

The situation is more complicated at the modified magnetosonic points, since no analytical condition is known. Our solution is a sequence of root finding routines to find the zeroes of specific functions. The method is almost the same at both MSP and MFP, therefore we will describe only the case of the MFP, highlighting the few differences when they exist.

Given the value of $\theta_{\mathrm{MFP}}$, we need to find the values of $M^{2}$ and $G^{2}$ that gives $\mathcal{N}_{1}=\mathcal{N}_{2}=\mathcal{D}=0$. In order to do this, we minimize the critical function

$$
\mathcal{C}_{\mathrm{F}}=\mathcal{N}_{2}^{2}+\mathcal{D}^{2}
$$

Even if it can be shown that if any two of $\mathcal{N}_{1}, \mathcal{N}_{2}, \mathcal{D}$ are zero, the third one must be zero as well, we found that for numerical reasons using $\mathcal{N}_{2}$ and $\mathcal{D}$ is more robust. We also found that at MSP it is better to use all the three numbers

$$
\mathcal{C}_{\mathrm{S}}=\mathcal{N}_{1}^{2}+\mathcal{N}_{2}^{2}+\mathcal{D}^{2}
$$

That has to do with the topology of the zeroes of the three numbers, which in the regimes we explored turn out to have deep narrow valleys running almost parallel to each other, so that it is difficult to find their intersection with enough precision.

In order to find the minimum of the critical function, we create a grid in the $M^{2}$ and $G^{2}$ space with boundaries dictated by the location of the Alfvén point. We then use the three points which have the lowest values of the critical function $\mathcal{C}$ as the starting nodes of a global simplectic minimising algorithm such as amebsa (see NR93, for a description). When the minimum has been found, we try to polish the solution with a further local powell minimising routine (NR93). At the end, we further verify that the absolute values of all the three numbers $\mathcal{N}_{1}, \mathcal{N}_{2}$ and $\mathcal{D}$ are close enough to zero $\left(i 10^{-10}\right)$. While this procedure of polishing the first solution and then cross checking again seems redundant and CPU consuming, it is our experience that this process avoids having spurious starting points which would contaminate the results of the next steps in our solution finding scheme.

Although we now know the initial values of $M^{2}$ and $G^{2}$, we cannot yet start the integration, since the derivative $d M^{2} / d \theta$ is still unknown: i.e. Eq. (1) would still be zero over zero. In order to recover the value of the derivative, we search the roots of another function. 
For a point $\theta$, very close to the critical point at $\theta_{\mathrm{MFP}}$, we can Taylor expand $G^{2}$ and $M^{2}$ to first order:

$$
\begin{gathered}
G^{2}\left(\theta_{\mathrm{MFP}}\right)=G^{2}(\theta)+\left.\frac{d G^{2}}{d \theta}\right|_{\theta}\left(\theta_{\mathrm{MFP}}-\theta\right) \\
M^{2}\left(\theta_{\mathrm{MFP}}\right)=M^{2}(\theta)+\left.\frac{d M^{2}}{d \theta}\right|_{\theta}\left(\theta_{\mathrm{MFP}}-\theta\right)
\end{gathered}
$$

Combining the two equations to eliminate $\theta_{\mathrm{MFP}}-\theta$, we define the following function

$$
Y\left(M^{2}(\theta)\right)=\left[M^{2}(\theta)-M^{2}\left(\theta_{\mathrm{MFP}}\right)\right]-\left[G^{2}(\theta)-G^{2}\left(\theta_{\mathrm{MFP}}\right)\right] \frac{\left.\frac{d M^{2}}{d \theta}\right|_{\theta}}{\left.\frac{d G^{2}}{d \theta}\right|_{\theta}}
$$

The final step is finding the zeroes of this function through an iterative method. Note that only $M^{2}(\theta)$ is a free variable. We approximate $G^{2}(\theta)-G^{2}\left(\theta_{\mathrm{MFP}}\right)=d G^{2} /\left.d \theta\right|_{\theta_{\mathrm{MFP}}}\left(\theta-\theta_{\mathrm{MFP}}\right)$. Once a guess of $M^{2}(\theta)$ is tried, we can calculate $d M^{2} / d \theta$ and $d G^{2} / d \theta$ at $\theta$ where the first one is not singular. At MFP we take $\theta=\theta_{\mathrm{MFP}}(1+\delta)$ and at MSP we take $\theta=\theta_{\mathrm{MSP}}(1-\delta)$, where $\delta=10^{-7}$

In most cases we find two roots for $M^{2}(\theta)$ : one smaller and one larger than $M^{2}\left(\theta_{\mathrm{MFP}}\right)$. Based on the physical picture of the accelerating jet and that $d M^{2} /\left.d \theta\right|_{A}<0$, we assume that the derivative is negative also at the critical points. Therefore at MFP we choose $M^{2}(\theta)<M^{2}\left(\theta_{\mathrm{MFP}}\right)$ and at MSP we take $M^{2}(\theta)>M^{2}\left(\theta_{\mathrm{MSP}}\right)$. We cannot justify this assumption based on general principles, and cannot provide a definitive proof, but we performed various tests allowing for the alternative choices, and never managed to find a solution. Now that we have the initial conditions we can integrate.

\section{APPENDIX D: CONVERSION TO PHYSICAL QUANTITIES}

When a solution is found, we convert and calculate all the relevant quantities in physical units to be comparable with observables. The conversions are taken from VK03. A few additional input quantities need to be provided in order to transform back a solution to physically measurable quantities. These are the magnetic field strength at the base, $B_{0}$, the ratio between radiation and matter pressure, $P_{\mathrm{M}} / P_{\mathrm{R}}$ (section 4, Eq. 29 in VK03) and the streamline label $\alpha \equiv \varpi^{2} / \varpi_{\mathrm{A}}^{2}$ (Section 2.1, Eq. 17 in VK03). The quantities $\alpha$ and $B_{0}$ provide the scaling for the given solution.

$$
\begin{aligned}
& \varpi=\varpi_{\mathrm{A}} G, \quad z=\frac{\varpi_{\mathrm{A}} G}{\tan (\theta)}, \quad \gamma=\frac{\mu^{\prime}}{\left(1-P_{\mathrm{g}}\right)} \frac{1}{\xi} \frac{\left(1-M^{2}-x_{\mathrm{A}}^{2}\right)}{\left(1-M^{2}-x^{2}\right)} \\
& T_{e}=\sqrt{x_{\mathrm{A}}^{2} \frac{B_{0}}{F \sigma_{\mathrm{M}}}}\left(\frac{(\xi-1)^{\Gamma /(\Gamma-1)} \alpha^{F-2}}{q\left(1+P_{\mathrm{M}} / P_{\mathrm{R}}\right)} \frac{3(\Gamma-1)}{4 \pi a \Gamma}\right)^{1 / 4}, \quad P_{\mathrm{M}} / P_{\mathrm{R}}=\left\{\begin{array}{ll}
0, & \theta_{\mathrm{i}} \leq 1 \\
1.85, & \theta_{\mathrm{i}} \geq 1
\end{array}, \quad \text { with } \quad \theta_{\mathrm{i}}=k T_{\mathrm{e}} / m_{\mathrm{e}} c^{2}\right. \\
& V_{\mathrm{p}}=-\frac{c F \sigma_{\mathrm{M}} M^{2} \sin (\theta)}{\gamma \xi x^{2} \cos (\psi+\theta)}, \quad V_{\phi}=\frac{c x_{\mathrm{A}}}{\gamma \xi G} \frac{\mu^{\prime}}{\left(1-P_{\mathrm{g}}\right)} \frac{G^{2}-M^{2}-x^{2}}{1-M^{2}-x^{2}}, \quad V_{\mathrm{tot}}=\sqrt{V_{\mathrm{p}}^{2}+V_{\phi}^{2}} \\
& B_{\mathrm{p}}=-\frac{B_{0} \sin (\theta) \alpha^{(F-2) / 2}}{G^{2} \cos (\psi+\theta)}, \quad B_{\phi}=-\frac{\mu^{\prime}}{\left(1-P_{\mathrm{g}}\right)} x_{\mathrm{A}}^{4} \frac{1-G^{2}}{1-M^{2}-x^{2}} \frac{B_{0} \alpha^{(F-2) / 2}}{F \sigma_{\mathrm{M}} x}, \quad B_{\mathrm{tot}}=\sqrt{B_{\mathrm{p}}^{2}+B_{\phi}^{2}} \\
& \rho_{0}=\frac{x_{\mathrm{A}}^{4} \xi B_{0}^{2} \alpha^{(F-2)}}{4 \pi M^{2}\left(c F \sigma_{\mathrm{M}}\right)^{2}}, \quad P=\frac{B_{0}^{2} \alpha^{(F-2)}}{4 \pi} \frac{\Gamma-1}{\Gamma} \frac{x_{\mathrm{A}}^{4}}{\left(F \sigma_{\mathrm{M}}\right)^{2}} \frac{\xi(\xi-1)}{M^{2}} \\
& h=\xi \gamma, \quad S=-\frac{x F \psi_{\mathrm{A}} B_{\phi}}{x_{\mathrm{A}}^{2} B_{0} \alpha^{(F-2) / 2}} \quad \mu^{\prime}=E_{t o t}=(h+S)\left(1-P_{\mathrm{g}}\right)=\mu\left(1-P_{\mathrm{g}}\right) \\
& \Omega=\frac{1}{\varpi}\left(V_{\phi}-B_{\phi} \frac{V_{\mathrm{p}}}{B_{\mathrm{p}}}\right)
\end{aligned}
$$

where $a$ is the Stefan-Boltzmann constant.

\section{REFERENCES}

Asada K., Nakamura M., 2012, ApJ, 745, L28

Asada K., Nakamura M., Doi A., Nagai H., Inoue M., 2014, ApJ, 781, L2

Avachat S. S., Perlman E. S., Adams S. C., Cara M., Owen F., Sparks W. B., Georganopoulos M., 2016, ApJ, 832, 3

Barniol Duran R., Tchekhovskoy A., Giannios D., 2016, preprint, (arXiv:1612.06929)

Belloni T. M., Motta S. E., 2016, Astrophysics of Black Holes: From Fundamental Aspects to Latest Developments, 440,61

Blandford R. D., Payne D. G., 1982, MNRAS, 199, 883

Bogovalov S., Tsinganos K., 1999, MNRAS, 305, 211

Cayatte V., Vlahakis N., Matsakos T., Lima J. J. G., Tsinganos K., Sauty C., 2014, ApJ, 788, L19

Connors R. M. T., et al., 2016, preprint, (arXiv:1612.00953)

Contopoulos J., 1994, ApJ, 432, 508 
Contopoulos J., 1995, ApJ, 450, 616

Corbel S., Fender R. P., 2002, ApJ, 573, L35

Crumley P., Ceccobello C., Connors R. M. T., Cavecchi Y., 2017, preprint, (arXiv:1703.02842)

Doeleman S. S., et al., 2008, Nature, 455, 78

Fabian A. C., 2012, ARA\&A, 50, 455

Falcke H., Körding E., Markoff S., 2004, A\&A, 414, 895

Fender R. P., 2003, MNRAS, 340, 1353

Fender R. P., Belloni T. M., Gallo E., 2004, MNRAS, 355, 1105

Fendt C., Ouyed R., 2004, The Astrophysical Journal, 608, 378

Feroz F., Hobson M. P., 2008, MNRAS, 384, 449

Feroz F., Hobson M. P., Bridges M., 2009, MNRAS, 398, 1601

Feroz F., Hobson M. P., Cameron E., Pettitt A. N., 2013, preprint, (arXiv:1306.2144)

Gallo E., Fender R., Kaiser C., Russell D., Morganti R., Oosterloo T., Heinz S., 2005, Nature, 436, 819

Giroletti M., et al., 2004, ApJ, 600, 127

Hada K., et al., 2016, ApJ, 817, 131

Harris D. E., Krawczynski H., 2006, ARA\&A, 44, 463

Hawley J. F., Krolik J. H., 2006, ApJ, 641, 103

Koide S., Shibata K., Kudoh T., Meier D. L., 2002, Science, 295, 1688

Komissarov S. S., Vlahakis N., Königl A., Barkov M. V., 2009, MNRAS, 394, 1182

Komissarov S. S., Vlahakis N., Königl A., 2010, MNRAS, 407, 17

Laurent P., Rodriguez J., Wilms J., Cadolle Bel M., Pottschmidt K., Grinberg V., 2011, Science, 332, 438

Li Z.-Y., Chiueh T., Begelman M. C., 1992, ApJ, 394, 459

Lovelace R. V. E., Contopoulos J., 1990, in Beck R., Wielebinski R., Kronberg P. P., eds, IAU Symposium Vol. 140, Galactic and Intergalactic Magnetic Fields. p. 337

Maitra D., Markoff S., Brocksopp C., Noble M., Nowak M., Wilms J., 2009, MNRAS, 398, 1638

Malzac J., 2014, Mon. Not. Roy. Astron. Soc., 443, 299

Markoff S., 2010, in Belloni T., ed., Lecture Notes in Physics, Berlin Springer Verlag Vol. 794, Lecture Notes in Physics, Berlin Springer Verlag. p. 143 (arXiv:0909.2574), doi:10.1007/978-3-540-76937-8*6

Markoff S., Falcke H., Fender R., 2001, A\&A, 372, L25

Markoff S., Nowak M. A., Wilms J., 2005, ApJ, 635, 1203

Martí-Vidal I., Muller S., Vlemmings W., Horellou C., Aalto S., 2015, Science, 348, 311

McKinney J. C., 2006, MNRAS, 368, 1561

Meier D. L., 2003, New Astron. Rev., 47, 667

Meier D. L., 2012, Black Hole Astrophysics: The Engine Paradigm

Mendoza S., Longair M. S., 2001, MNRAS, 324, 149

Merloni A., Heinz S., di Matteo T., 2003, MNRAS, 345, 1057

Mertens F., Lobanov A. P., Walker R. C., Hardee P. E., 2016, A\&A, 595, A54

Meyer E. T., Sparks W. B., Biretta J. A., Anderson J., Sohn S. T., van der Marel R. P., Norman C., Nakamura M., 2013, ApJ, 774, L21

Michel F. C., 1969, ApJ, 158, 727

Migliari S., Tomsick J. A., Maccarone T. J., Gallo E., Fender R. P., Nelemans G., Russell D. M., 2006, ApJ, 643, L41

Miller-Jones J. C. A., Fender R. P., Nakar E., 2006, MNRAS, 367, 1432

Mościbrodzka M., Falcke H., Shiokawa H., 2016, A\&A, 586, A38

Nakamura M., Asada K., 2013, ApJ, 775, 118

Nemmen R. S., Tchekhovskoy A., 2015, MNRAS, 449, 316

Parker E. N., 1958, ApJ, 128, 664

Pepe C., Vila G. S., Romero G. E., 2015, A\&A, 584, A95

Perlman E. S., Biretta J. A., Zhou F., Sparks W. B., Macchetto F. D., 1999, AJ, 117, 2185

Polko P., Meier D. L., Markoff S., 2010, ApJ, 723, 1343

Polko P., Meier D. L., Markoff S., 2013, MNRAS, 428, 587

Polko P., Meier D. L., Markoff S., 2014, MNRAS, 438, 959

Potter W. J., Cotter G., 2012, MNRAS, 423, 756

Press W. H., Teukolsky S. A., Vetterling W. T., Flannery B. P., 1993, Numerical Recipes in FORTRAN; The Art of Scientific Computing, 2nd edn. Cambridge University Press, New York, NY, USA

Prieto M. A., Fernández-Ontiveros J. A., Markoff S., Espada D., González-Martín O., 2016, MNRAS, 457, 3801

Rawlings S., Saunders R., 1991, Nature, 349, 138

Rees M. J., Meszaros P., 1994, ApJ, 430, L93

Ressler S. M., Tchekhovskoy A., Quataert E., Chandra M., Gammie C. F., 2015, MNRAS, 454, 1848

Romero G. E., Torres D. F., Kaufman Bernadó M. M., Mirabel I. F., 2003, A\&A, 410, L1

Romero G. E., Boettcher M., Markoff S., Tavecchio F., 2017, Space Sci. Rev.,

Russell D. M., Shahbaz T., 2014, MNRAS, 438, 2083

Russell T. D., Soria R., Miller-Jones J. C. A., Curran P. A., Markoff S., Russell D. M., Sivakoff G. R., 2014, MNRAS, 439, 1390

Russell H. R., Fabian A. C., McNamara B. R., Broderick A. E., 2015, MNRAS, 451, 588

Sauty C., Tsinganos K., 1994, A\&A, 287, 893

Sauty C., Cayatte V., Lima J. J. G., Matsakos T., Tsinganos K., 2012, ApJ, 759, L1

Stoer J., Bulirsch R., 2013, Introduction to numerical analysis. Vol. 12, Springer Science \& Business Media

Tchekhovskoy A., Bromberg O., 2016, MNRAS, 461, L46

Tchekhovskoy A., Narayan R., McKinney J. C., 2011, MNRAS, 418, L79

Vlahakis N., Königl A., 2003, ApJ, 596, 1080 
Vlahakis N., Tsinganos K., Sauty C., Trussoni E., 2000, MNRAS, 318, 417

Weber E. J., Davis Jr. L., 1967, ApJ, 148, 217

Weisskopf M. C., et al., 2016, in Space Telescopes and Instrumentation 2016: Ultraviolet to Gamma Ray. p. 990517, doi: $10.1117 / 12.2235240$

Yuan F., Cui W., Narayan R., 2005, ApJ, 620, 905

Zdziarski A. A., Lubiński P., Sikora M., 2012, MNRAS, 423, 663

Zdziarski A. A., Stawarz Ł., Pjanka P., Sikora M., 2014, MNRAS, 440, 2238 